\title{
A Finite Element Study of Double Diffusive Mixed Convection in a Concentration Stratified Darcian Fluid Saturated Porous Enclosure under Injection/Suction Effect
}

\author{
B. V. Rathish Kumar' ${ }^{1}$ and S. V. S. S. N. V. G. Krishna Murthy ${ }^{2}$ \\ ${ }^{1}$ Department of Mathematics and Statistics, Indian Institute of Technology Kanpur, Kanpur 208016, India \\ 2 Department of Applied Mathematics, Defence Institute of Advanced Technology, Pune 411025, India
}

Correspondence should be addressed to B. V. Rathish Kumar, drbvrk@gmail.com

Received 9 April 2012; Accepted 23 April 2012

Academic Editor: Mehmet Sezer

Copyright (c) 2012 B. V. R. Kumar and S. V. S. S. N. V. G. Krishna Murthy. This is an open access article distributed under the Creative Commons Attribution License, which permits unrestricted use, distribution, and reproduction in any medium, provided the original work is properly cited.

Numerical investigation of mixed convection flow in a concentration-stratified fluid-saturated vertical square porous enclosure is investigated by Galerkin finite element method. The forced flow conditions are imposed by providing an inlet at the bottom wall and an outlet with a suction on the top wall. The free convection is induced by introducing a hot but isothermal temperature on the left vertical wall together with Boussinesq approximation on density. Numerical results are presented by tracing the cumulative heat and mass fluxes, streamline and isotherms of the fluid for a wide range of governing parameters such as suction/injection velocity " $a$ ", suction/injection width $(D / H)$, mass stratification parameters $\left(S_{C}\right)$, Rayleigh number "Ra", buoyancy ratio " $B$ ", and Lewis number "Le".

\section{Introduction}

A wide range of scientific and engineering applications such as packed bed reactors, porous insulation, beds of fossil fuels, nuclear waste disposal, usage of porous conical bearings in lubrication technology, fibrous insulation systems, grain storage, chemical catalytic reactors, solar collectors, heat exchangers, food processing, energy efficient drying process, geophysics and energy related problems, requires a detailed understanding of convective heat transfer process in a fluid-saturated porous media. These applications are reviewed in recent books by [1-6], provided a detailed coverage of research works related to convective heat transfer in Porous media. In particular applications such as cooling of radioactive waste containers, solar power collectors, electronic equipment cooling, thermal insulation of buildings, have drawn considerable attention in last several years onto flow through porous enclosures. Some of the industrial technical processes like solar central receivers exposed to wind currents, electronic 
devices cooled by fans, heat exchangers, microelectronic devices packed with micro-pin-fin, vented enclosures filled with microspheres require a detailed study of mixed convection process in porous enclosures under different conditions.

Mixed convection is a fundamentally significant heat transfer mechanism that occurs in a selection of industrial and technological applications. For example, it is of great interest to thermal designers in the field of electronic cooling. Given that its occurrence is frequent, there is a necessity to understand the physics of this fundamental mechanism at board level. It is motivated both by a desire to gain an understanding the fundamental physics of mixed convection and to solve design problems in scientific and electronic applications where mixed convection often occurs. Apart from this situation there are many more industrial and natural problems like microwave heating of dielectric materials using a rectangular wave guide, sintered porous heat sink for cooling of high-powered microprocessors for its applications, biomass growth on the hydraulic properties of saturated porous media, that is, bioclogging and so forth, where a thorough understanding of mixed convection process is essential. Experimental studies need to be supplemented by theoretical support. This certainly calls for a complete theoretical investigation of mixed convection process in porous media.

A comprehensive review of several analytical, numerical, and experimental results has been published on mixed convection process in porous enclosures that are reported in the literature. Reference [7] has numerically studied mixed convection in a vented enclosure with continuum fluid and isothermal vertical surface. Bansod and Jadhav [8] presented the mixed convection heat and mass transfer near a vertical surface in a stratified porous medium using an integral method. Chamkha $[9,10]$ in his mathematical model had additionally included transverse magnetic effects too. W. J. Chang and W. L. Chang [11] looked at mixed convection in a vertical tube partially filled with porous medium. Chen [12], Chou and Chung [13], and Prasad et al. [14] have analytically analyzed mixed convection with the free convection induced by either a horizontal or a vertical hot plate enclosed in a semiinfinite porous medium under boundary layer assumptions. C. K. Chen and C. H. Chen [15] have analyzed the non-Darcian and nonuniform porosity effects on conjugate mixed convection heat transfer from a plate fin in porous media under boundary layer assumptions, employing one-dimensional heat conduction equation for closing the conjugate heat transfer model. El-Amin et al. [16] numerically investigated the interaction of mixed convection with thermal radiation of an optical dense viscous fluid adjacent to an isothermal cone imbedded in a porous medium with Rossel and diffusion approximation incorporating the variation of permeability and thermal conductivity. Elbashbeshy [17] solved the problem of mixed convection along a vertical plate embedded in a non-Darcian porous medium with suction and injection under boundary layer assumptions using similar variables defined based on scale analysis.

Hassanien and Al-arabi [18] have simplified the boundary layer equations using scale analysis and numerically solved the problem of non-Darcian unsteady mixed convection flow near the stagnation point on heated vertical surface embedded in a porous medium with thermal radiation, and variable viscosity has been considered. Jayanthi and Kumari [19] have analytically studied the effect of variable viscosity on non-Darcian free or mixed convection flow on a vertical surface in a non-Newtonian fluid saturated porous medium. Khanafer and Vafai [20] investigated the double diffusive mixed convection in a square enclosure filled with a porous medium in the presence of moving boundary which was formulated and solved numerically by finite-volume approach along with the alternating direction implicit scheme. Recently, Wong and Saied [21] have studied numerically the non-Darcian effects, based on Brinkman-Forchheimer-extended Darcy model, on jet impingement cooling in a horizontal 
porous layer in a mixed convection regime. They have employed finite volume method in conjunction with power-law scheme for convection-diffusion model. Lai [22] explained in detail about the mixed convection in saturated porous media. Kumar et al. [23] have studied mixed convection flow in a vertical square enclosure filled with a non-Darcian fluid saturated homogeneous porous medium by providing an inlet at the bottom wall and an outlet with a suction on the top wall numerically by using the Galerkin finite element method. Nazar et al. [24] analyzed mixed convection boundary layer flow past a horizontal circular cylinder in a porous medium based on Brinkman model.

Singh et al. [25] reported on mixed convection process in a flow past a porous vertical plate bounded by a porous medium in a rotating system in the presence of magnetic filed. Shohel Mahmud and Pop [26] have numerically extended the study of [7] to Darcian porous square vented enclosure using finite volume approach. Srinivasacharya and RamReddy [27] have analysed the effects of thermal and solutal stratification on mixed convection along a vertical plate embedded in a micropolar fluid saturated non-Darcy porous medium. kurdyumov and Linan [28] presented an analysis for the steady, two-dimensional, free convection around line sources of heat and heated cylinders in unbounded saturated porous media. Vafai and Hadim [29] have presented an overview on the computational studies of heat transfer in porous media both under natural and mixed convection processes. Waheed et al. [30] have studied numerically the buoyancy- and the shear-driven flow induced by a hot plate moving through the horizontal midplane of a rectangular enclosure filled with fluid-saturated porous medium.

So far not much work has been reported on Darcian mixed convection in vertical square porous enclosure. In particular mixed convection in a vertical porous enclosure under simultaneous suction/injection effects on opposite walls, which becomes very relevant in the context of electronic devices, environmental chamber for bacterial culture preservation, and so forth, has not been considered so far. In the present study, attention will be focused on a new problem of steady mixed convection heat and mass transfer with concentration stratification inside a vertical square fluid saturated porous enclosure with fluid injection at the bottom wall and a suction at the top wall. Thus forced convection is imposed by this combination of suction/injection flow conditions. The free convection is induced by the hot and isothermal left vertical wall. Detailed numerical simulations are carried out by Galerkin finite element method for a wide range of parameters such as Rayleigh number "Ra", suction/injection flow speed " $a$ ", suction/injction width $(D / H)$, mass stratification parameters $\left(S_{C}\right)$, buoyancy ratio " $B$ ", Lewis number "Le". Flow and temperature distribution is analyzed by tracing streamlines, isotherms, local/cumulative heat fluxes.

\section{Mathematical Formulation}

We consider a two-dimensional square enclosure filled with a fluid saturated porous medium with the left vertical wall at the uniform temperature $t_{w}$ and the surface concentration as $c_{w}$ which are assumed to be higher than the ambient temperature and concentration, respectively, and the right vertical wall is kept at uniform ambient temperature $t_{0}$ and with the ambient species concentration as $c_{\infty, y}$ is defined as and shown in Figure 1:

$$
c_{\infty, y}=c_{\infty, 0}+s_{c} y ; \quad \text { where } s_{c}=\frac{d c_{\infty, y}}{d y}
$$

other two walls are adiabatic, excluding the inlet/outlet portion at the bottom/top wall. The other two walls are excluding the suction/injection windows, which are permeable 


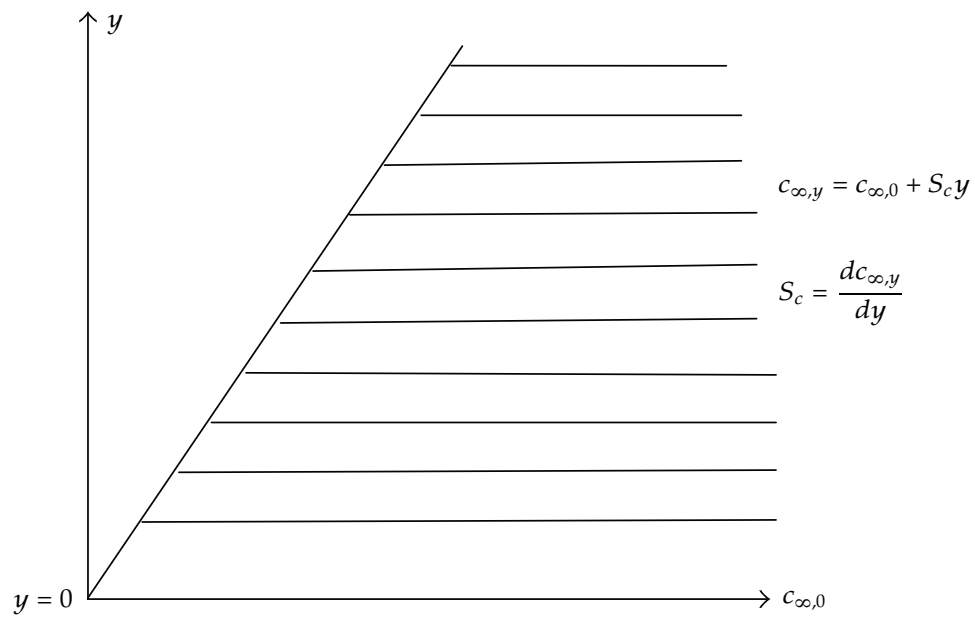

Figure 1: Model with concentration stratification.



Figure 2: Schematic diagram of the porous enclosure with prescribed boundary conditions.

with zero normal thermal gradients. Here the forced flow conditions are brought in by the combination of suction and injection effects on bottom and top walls, respectively. The inflow is considered to be at ambient temperature. $D$ is the width of the inlet and outlet and $H$ is the height of the cavity. The porous medium is assumed to be isotropic with permeability $K$, and Boussinesq approximation is valid in the momentum equation for the density term. Dispersion effect has been neglected. $x$ and $y$ are the horizontal and vertical components, respectively. The physical configuration and the coordinate system used in the current study are shown in Figure 2. The equations governing the steady-state double diffusive Darcy mixed convection flow along a heated vertical wall under concentration stratification with the above mentioned assumptions, nondimensional equations [31-35], governing the flow, 
heat, and mass transport in a fluid saturated porous media, can be written in terms of the following $\Psi-T-C$ nondimensional governing equations:

$$
\begin{gathered}
\frac{\partial^{2} \Psi}{\partial X^{2}}+\frac{\partial^{2} \Psi}{\partial Y^{2}}=\frac{\partial T}{\partial X}+B \frac{\partial C}{\partial X^{\prime}} \\
\frac{\partial \Psi}{\partial Y} \frac{\partial T}{\partial X}-\frac{\partial \Psi}{\partial X} \frac{\partial T}{\partial Y}=\frac{1}{R a}\left(\frac{\partial^{2} T}{\partial X^{2}}+\frac{\partial^{2} T}{\partial Y^{2}}\right), \\
\frac{\partial \Psi}{\partial Y} \frac{\partial C}{\partial X}+S_{C} \frac{\partial \Psi}{\partial Y}-\frac{\partial \Psi}{\partial X} \frac{\partial C}{\partial Y}=\frac{1}{\operatorname{Ra~Le}\left(\frac{\partial^{2} C}{\partial X^{2}}+\frac{\partial^{2} C}{\partial Y^{2}}\right),}
\end{gathered}
$$

with the following boundary conditions:

left wall: $\Psi=0, \quad T=1, \quad C=1-S_{C} Y$ on $X=0,0 \leq Y \leq 1$,

right wall: $\Psi=0, \quad T=0, \quad C=0$ on $X=1,0 \leq Y \leq 1$,

permeable bottom wall:

$\frac{\partial \Psi}{\partial Y}=0, \quad \frac{\partial T}{\partial Y}=0, \quad \frac{\partial C}{\partial Y}=0 \quad$ at $0 \leq X \leq X_{1}, \quad X_{1}+\left(\frac{D}{H}\right) \leq X \leq 1$, on $Y=0$,

permeable top wall:

$\frac{\partial \Psi}{\partial Y}=0, \quad \frac{\partial T}{\partial Y}=0, \quad \frac{\partial C}{\partial Y}=0 \quad$ at $0 \leq X \leq X_{2}, \quad X_{2}+\left(\frac{D}{H}\right) \leq X \leq 1$, on $Y=1$,

injection window: $\Psi=-a X, \quad T=0, \quad C=0 \quad$ at $X_{1} \leq X \leq X_{1}+\left(\frac{D}{H}\right)$, on $Y=0$,

suction window: $\Psi=-a X, \quad \frac{\partial T}{\partial Y}=0, \quad \frac{\partial C}{\partial Y}=0 \quad$ at $X_{2} \leq X \leq X_{2}+\left(\frac{D}{H}\right)$, on $Y=1$.

The nondimensional variables are defined as follows:

$$
\begin{gathered}
X=\frac{x}{H}, \quad Y=\frac{y}{H}, \quad U=\frac{u}{V_{c}}, \quad V=\frac{v}{V_{c}}, \quad a=\frac{V_{0}}{V_{c}}, \\
\text { where } V_{c}=\frac{K g \beta \Delta t}{v}, \quad S_{C}=\frac{1}{\Delta c} \frac{d c_{\infty, y}}{d Y}, \\
C=\frac{c-c_{\infty, x}}{c_{w}-c_{\infty}, 0}, \quad B=\frac{\beta_{c}\left(c_{w}-c_{\infty}, 0\right)}{\beta_{t}\left(t_{w}-t_{0}\right)}, \quad \mathrm{Le}=\frac{\alpha}{d}, \quad T=\frac{t-t_{0}}{t_{w}-t_{0}}, \quad \mathrm{Ra}=\frac{K g \beta H \Delta t}{v \alpha}, \\
\text { where } \Delta t=t_{w}-t_{0} .
\end{gathered}
$$


The nondimensional stream function $\Psi$ is defined as

$$
U=\frac{\partial \Psi}{\partial Y}, \quad V=-\frac{\partial \Psi}{\partial X}
$$

The local and cumulative heat flux $\left(Q H_{x}\right)$ along the left vertical wall is computed by the relation for Darcy model:

$$
\mathrm{Nu}_{X}=\left(-\frac{\partial T}{\partial X}\right)_{X=0}, \quad \operatorname{CHFLX}=Q_{\xi}=\int_{0}^{\xi}\left(-\frac{\partial T}{\partial X}\right)_{X=0} d Y, \quad 0 \leq \xi \leq 1 .
$$

Similarly, the local and cumulative mass flux $\left(Q M_{X}\right)$ along the vertical wall is given by the relation for Darcy model:

$$
Q m_{X}=\left(-\frac{\partial C}{\partial X}\right)_{X=0}, \quad \text { CMFLX }=Q_{\xi}=\int_{0}^{\xi}\left(-\frac{\partial C}{\partial X}\right)_{X=0} d Y, \quad 0 \leq \xi \leq 1,
$$

where $\xi$ is the running coordinate in $Y$ direction and the upper limits of the integration, that is, $\xi=1$ in the above two expressions, give the global heat flux or Nusselt number $(\mathrm{Nu})$ and global mass flux or Sherwood number (Sh), respectively.

\section{Solution Methodology: Finite Element Formulation}

Governing equations (2.2) together with boundary conditions (2.3) has been solved using Galerkin finite element method. Following is the outline of finite element formulation used in the current study. Let $\Omega$ denote the domain of interest and $\Gamma$ let be the boundary of the domain. The discretized representation of $\Omega$ is given by $\bar{\Omega}=\bigcup_{e=1}^{N E L} \Omega^{e}$, where $\Omega^{e}$ denotes a typical bilinear element of the discretized domain and NEL is the total number of such elements. The discretized elements are fully disjoint, that is, $\bigcap_{e} \Omega^{e}=\{\}$. The discretized representation of the field variables $\Psi, T, C$ on a typical bilinear element $\Omega^{e}$ is

$$
\Psi=\sum_{i=1}^{4} \Psi_{i}^{e} N_{i}^{e}, \quad T=\sum_{i=1}^{4} T_{i}^{e} N_{i}^{e}, \quad C=\sum_{i=1}^{4} C_{i}^{e} N_{i}^{e},
$$

where $N_{i}^{e}$ denotes the standard bilinear interpolation function on a typical element $\Omega^{e}$.

Consider the Galerkin weighted residual forms of governing equation (2.2) on $\Omega^{e}$ :

$$
\begin{aligned}
& \int_{\Omega^{e}} W\left\{\left(\frac{\partial^{2} \Psi}{\partial X^{2}}+\frac{\partial^{2} \Psi}{\partial Y^{2}}\right)-\left(\frac{\partial T}{\partial X}+B \frac{\partial C}{\partial X}\right)\right\} d \Omega^{e}=0, \\
& \int_{\Omega^{e}} W\left\{\left(\frac{\partial \Psi}{\partial Y} \frac{\partial T}{\partial X}-\frac{\partial \Psi}{\partial X} \frac{\partial T}{\partial Y}\right)-\frac{1}{R a}\left(\frac{\partial^{2} T}{\partial X^{2}}+\frac{\partial^{2} T}{\partial Y^{2}}\right)\right\} d \Omega^{e}=0, \\
& \int_{\Omega^{e}} W\left\{\left(\frac{\partial \Psi}{\partial Y} \frac{\partial C}{\partial X}+S_{C} \frac{\partial \Psi}{\partial Y}-\frac{\partial \Psi}{\partial X} \frac{\partial C}{\partial Y}\right)-\frac{1}{\operatorname{RaLe}}\left(\frac{\partial^{2} C}{\partial X^{2}}+\frac{\partial^{2} C}{\partial Y^{2}}\right)\right\} d \Omega^{e}=0
\end{aligned}
$$


Here, $W$ is the weight function that is chosen as $N_{i}$ equal to the interpolation function. Rewriting (3.2) in the weak form and on introducing the element-level discretized representation for the field variables, that is, (3.1), one would arrive at the following element level matrix equation:

$$
M^{e} a^{e}=f^{e}
$$

where

$$
M_{i j k}=\left[\begin{array}{ccc}
A_{i j}^{11} & A_{i j}^{12} & A_{i j}^{13} \\
0 & A_{i j k}^{22} & 0 \\
A_{i j}^{31} & 0 & A_{i j k}^{33}
\end{array}\right], \quad a_{j}^{e}=\left[\begin{array}{lll}
\Psi_{j}^{e} & T_{j}^{e} & C_{j}^{e}
\end{array}\right]^{T}, \quad f_{i}^{e}=\left[\begin{array}{lll}
f_{i_{1}}^{e} & f_{i_{2}}^{e} & f_{i_{3}}^{e}
\end{array}\right]^{T} .
$$

Here,

$$
\begin{gathered}
A_{i j}^{11}=\int_{\Omega^{e}}\left(\frac{\partial N_{i}^{e}}{\partial X} \frac{\partial N_{j}^{e}}{\partial X}+\frac{\partial N_{i}^{e}}{\partial Y} \frac{\partial N_{j}^{e}}{\partial Y}\right) d \Omega^{e}, \\
A_{i j}^{12}=\int_{\Omega^{e}} N_{i}^{e} \frac{\partial N_{j}^{e}}{\partial X} d \Omega^{e}, \\
A_{i j}^{13}=\int_{\Omega^{e}} B N_{i}^{e} \frac{\partial N_{j}^{e}}{\partial X} d \Omega^{e}, \\
A_{i j k}^{22}=\int_{\Omega^{e}}\left[N_{i}^{e}\left\{\sum_{k=1}^{4}\left(\frac{\partial N_{j}^{e}}{\partial X} \frac{\partial N_{k}^{e}}{\partial Y}-\frac{\partial N_{j}^{e}}{\partial Y} \frac{\partial N_{k}^{e}}{\partial X}\right) \Psi_{k}^{e}\right\}+\frac{1}{\operatorname{Ra}}\left(\frac{\partial N_{i}^{e}}{\partial X} \frac{\partial N_{j}^{e}}{\partial X}+\frac{\partial N_{i}^{e}}{\partial Y} \frac{\partial N_{j}^{e}}{\partial Y}\right)\right] d \Omega^{e}, \\
A_{i j k}^{33}=\int_{\Omega^{e}}\left[N_{i}^{e}\left\{\sum_{k=1}^{41}\left(\frac{\partial N_{j}^{e}}{\partial X} \frac{\partial N_{k}^{e}}{\partial Y}-\frac{\partial N_{j}^{e}}{\partial Y} \frac{\partial N_{k}^{e}}{\partial X}\right) \Psi_{i}^{e} \frac{\partial N_{j}^{e}}{\partial Y} d \Omega^{e}, \frac{1}{\operatorname{Ra} L e}\left(\frac{\partial N_{i}^{e}}{\partial X} \frac{\partial N_{j}^{e}}{\partial X}+\frac{\partial N_{i}^{e}}{\partial Y} \frac{\partial N_{j}^{e}}{\partial Y}\right)\right] d \Omega^{e},\right. \\
f_{i_{2}}^{e}=\frac{1}{\operatorname{Ra}} \int_{\Gamma^{e}} N_{i}^{e} \frac{\partial T}{\partial n} d \Gamma^{e}, \\
f_{i_{3}}^{e}=\frac{1}{\operatorname{Ra} L_{i^{e}}} \int_{\Gamma^{e}} N_{i}^{e} \frac{\partial C}{\partial n} d \Gamma^{e} .
\end{gathered}
$$

Here, $n$ denotes the normal to the boundary of the porous cavity. The nonlinear global system obtained by assembling the local element matrix (3.3) is solved iteratively using frontal method for nonlinear systems to an accuracy of $\epsilon=10^{-4}$ on the relative error of nodal field variables from successive iterations, that is, $\left|\xi_{i}^{s+1}-\xi_{i}^{s}\right| \leq \epsilon$, where $\xi_{i}^{s}=\Psi_{i}^{s}$ or $T_{i}^{s}$ or $C_{i}^{s}$. Here the 


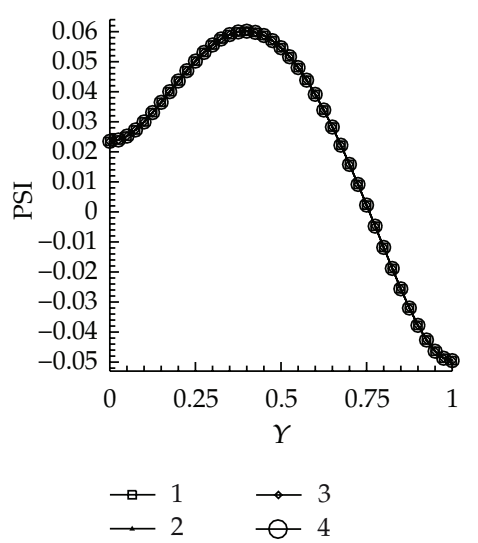

(a)



(d)

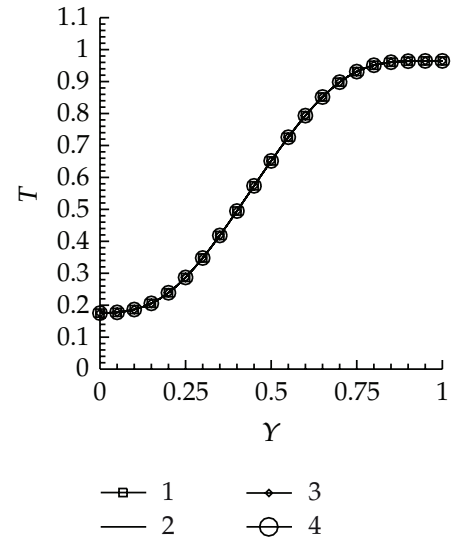

(b)

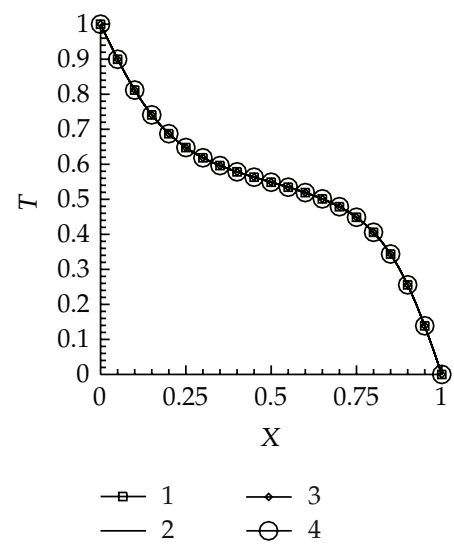

(e)

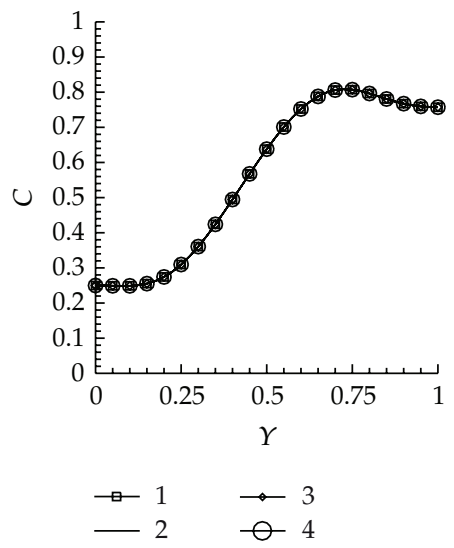

(c)

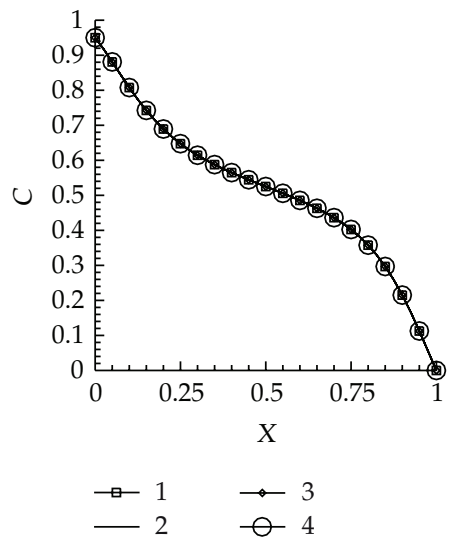

(f)

Figure 3: Grid selection results on different grid systems ((1) $21 \times 21$, (2) $31 \times 31$, (3) $41 \times 41$, (4) $51 \times 51$ ) for (a) stream function, (b) temperature, and (c) concentration values when $a=0.5, \operatorname{Ra}=100,(D / H)=S_{C}=$ 0.1 , Le $=1, B=1$ along $X$-axis at fixed $Y=0.5$, and the corresponding plots along $Y$-axis at fixed $X=0.5$ are in (d-f), respectively.

superscript $s$ refers to the iteration level and $i$ refers to the nodal point index. To accelerate the convergence, we underrelax the results from successive iteration by a factor of 0.25 .

\section{Results and Discussion}

The parameters which influence the double diffusive natural convection process in the present study are-buoyancy ratio " $B$ ", Lewis number "Le", mass stratification parameters $\left(S_{C}\right)$, injection/suction $(I / S)$ velocity parameter " $a$ ", $I / S$ window width $(D / H)$, and Rayleigh number "Ra". Grid selection tests have been carried out on five different grid systems consisting of $21 \times 21,31 \times 31,41 \times 41,51 \times 51$, and $61 \times 61$ elements. On these five grid systems simulations have been carried out for various combinations of above-mentioned parameters, and obtained stream function, temperature, and concentration values are compared. Few of these comparison plots are presented in Figure 3. In Figures 3(a)-3(c) $\Psi$, $T, C$ values are plotted along $X$-axis at fixed $Y=0.5$ for $a=0.5, \operatorname{Ra}=100,(D / H)=S_{C}=0.1$, 




Figure 4: Cumulative heat flux plots along the left vertical wall while fixing $\operatorname{Le}=1, B=1,(D / H)=0.1$, $\mathrm{Ra}=100, S_{C}=0.1$ for different values of " $a$ ".

Table 1: Nusselt number values for grid validation.

\begin{tabular}{lc}
\hline Grid size & Nusselt number \\
\hline $21 \times 21$ & 2.184652 \\
$31 \times 31$ & 2.1899673 \\
$41 \times 41$ & 2.19294511 \\
$51 \times 51$ & 2.19305301 \\
$61 \times 61$ & 2.19305101 \\
\hline
\end{tabular}

Le $=1, B=1$, and the corresponding plots along $Y$-axis at fixed $X=0.5$ are in Figures 3(d)$3(\mathrm{f})$, respectively. It is observed from these plots and the corresponding $\mathrm{Nu}$ data (Table 1 ) that as one moves from $51 \times 51$ to higher grid systems, there is only a marginal change in the solutions. Hence $51 \times 51$ grid system has been chosen for extensive numerical simulations. Details of the study showing the influence of various parameters on heat and mass transfer process in the presence of concentration stratification are given in the following subsections.

\subsection{Influence of $I / S$ Parameter " $a$ "}

The influence of $I / S$ velocity parameter " $a$ " on the heat transfer process in the presence of concentration stratification has been analyzed by comparing the cumulative heat fluxes (CHFLXs) along the left vertical wall of the porous enclosure. In Figure 4 CHFLXs along the left vertical wall are presented for $0 \leq a \leq 0.5$, Le $=1, B=1,(D / H)=0.1, \mathrm{Ra}=100, S_{C}=$ 0.1 . At all values of " $a$ ", CHFLXs are seen to increase as one moves along left vertical wall, 


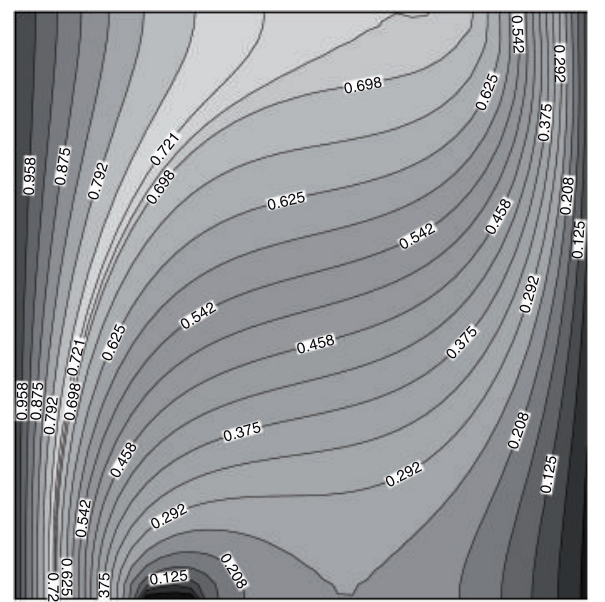

(a)

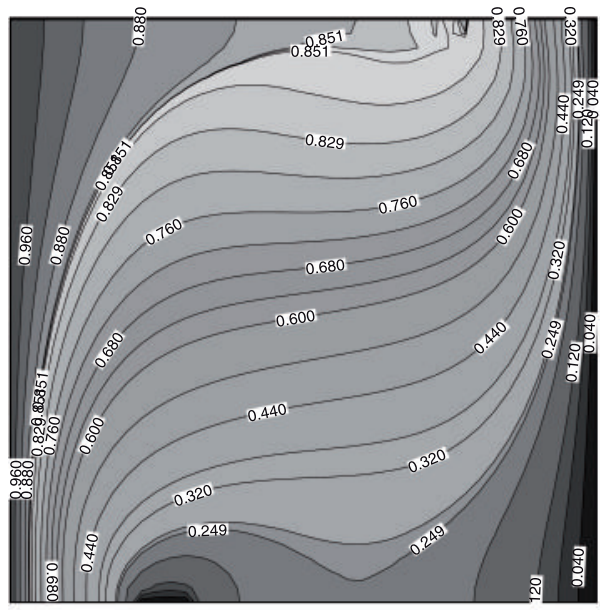

(c)

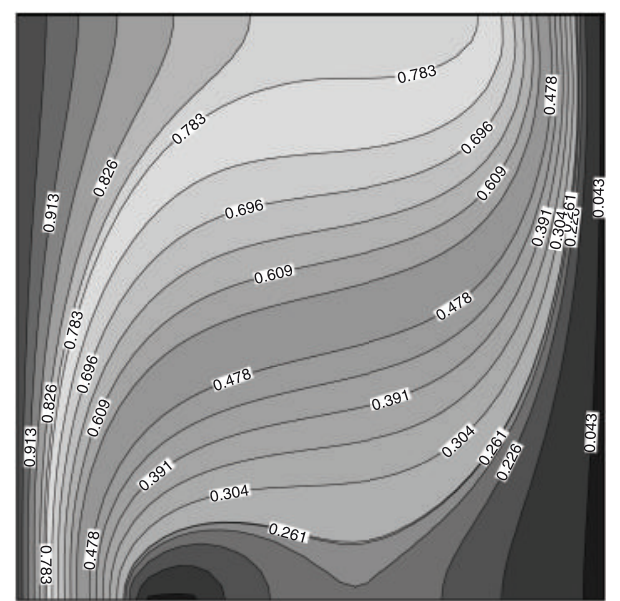

(b)



(d)

Figure 5: Isotherm contours along the left vertical wall while fixing $\operatorname{Le}=1, B=1,(D / H)=0.1, \mathrm{Ra}=100$, $S_{C}=0.1$ for (a) $a=0.0$, (b) $a=0.1$, (c) $a=0.25$, (d) $a=0.5$.

that is, $0 \leq Y \leq 1$. This increase is relatively sharp along the leading half portion of the left vertical wall, that is, $0 \leq Y \leq 0.65$. In this region CHFLXs are seen to increase with increasing values of " $a$ ". However along $0.65 \leq Y \leq 1$, CHFLXs are seen to decrease with increasing " $a$ ". Consequently the global heat flux (GHFLX) or Nusselt number (Nu) is seen to decrease with increasing values of " $a$ ". To explain these observations one has to investigate the temperature and flow fields. In Figures 5(a)-5(d) and Figures 6(a)-6(d) isotherms and streamlines corresponding to the above set of parameters are traced.

From the isotherm plots in Figures 5(a)-5(d) one can notice the manifestation of sharp thermal boundary layer (TBL) along the left vertical wall. While, for the case $a=0$, the TBLs continue to remain sharp all along the left wall, those corresponding to $a>0$ tend to get increasing blunt for $Y>0.65$ as " $a$ " increases. From the isotherm values one can clearly notice that the introduction of forced convection has led to greater transfer into the core of 


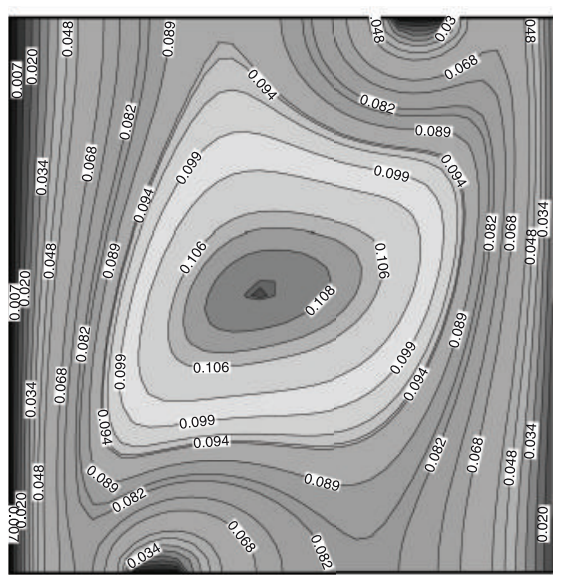

(a)

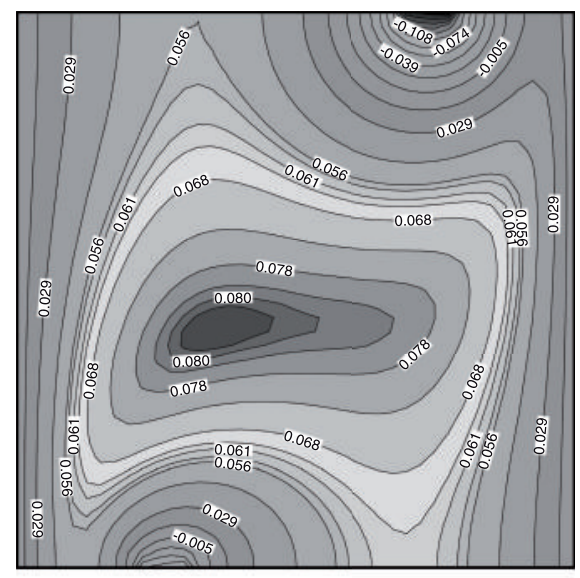

(c)

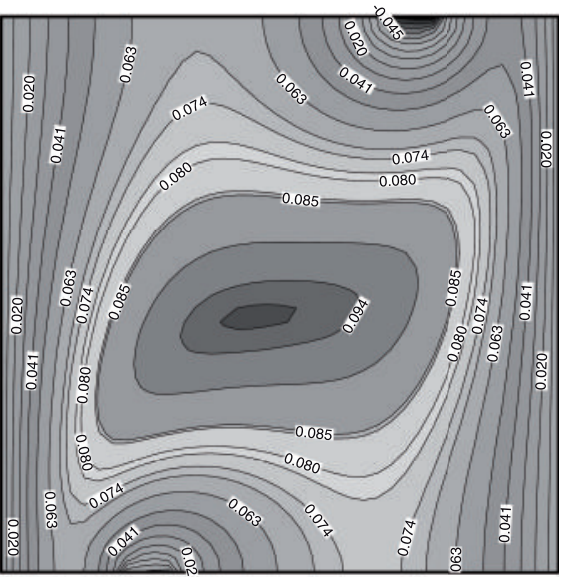

(b)

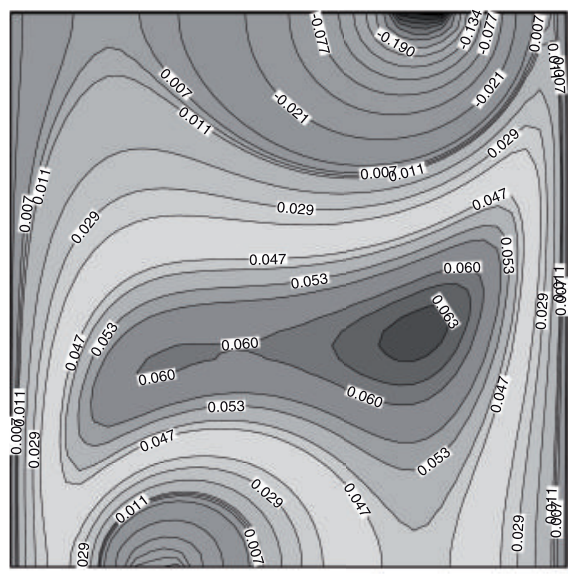

(d)

Figure 6: Streamline contours along the left vertical wall while fixing $\operatorname{Le}=1, B=1,(D / H)=0.1, \mathrm{Ra}=100$, $S_{C}=0.1$ for (a) $a=0.0$, (b) $a=0.1$, (c) $a=0.25$, (d) $a=0.5$.

the domain. Clearly it indicates a raise in average temperature in the porous enclosure with increasing values of $I / S$ velocity. With increasing " $a$ " one can also notice an increase in the transverse temperature gradients in the core of the domain.

The streamline plots in Figures 6(a)-6(d) depict the manifestation of a dominant primary circulation zone in the core of the domain and secondary circulation patterns centered on the $I / S$ windows. On increasing the $I / S$ velocity while the primary circulation zone undergoes a dominant longitudinal stretch, which nearly touches both the vertical walls, the secondary zones are seen to increasingly swell and dominantly impinge onto the primary circulation zone. Clearly the longitudinal stretch in primary circulation zone leads to better mixing of hot and cold fluids thereby leading to better heat transfer from the hot left vertical wall into the porous enclosure. Clearly the increase in the forced convection component leads to better heating in the porous enclosure and an overall raise in temperature of the porous enclosure. Hence the observed fall in the global cumulative heat flux (GCHLX) with increasing " $a$ " turns out to be natural consequence. 


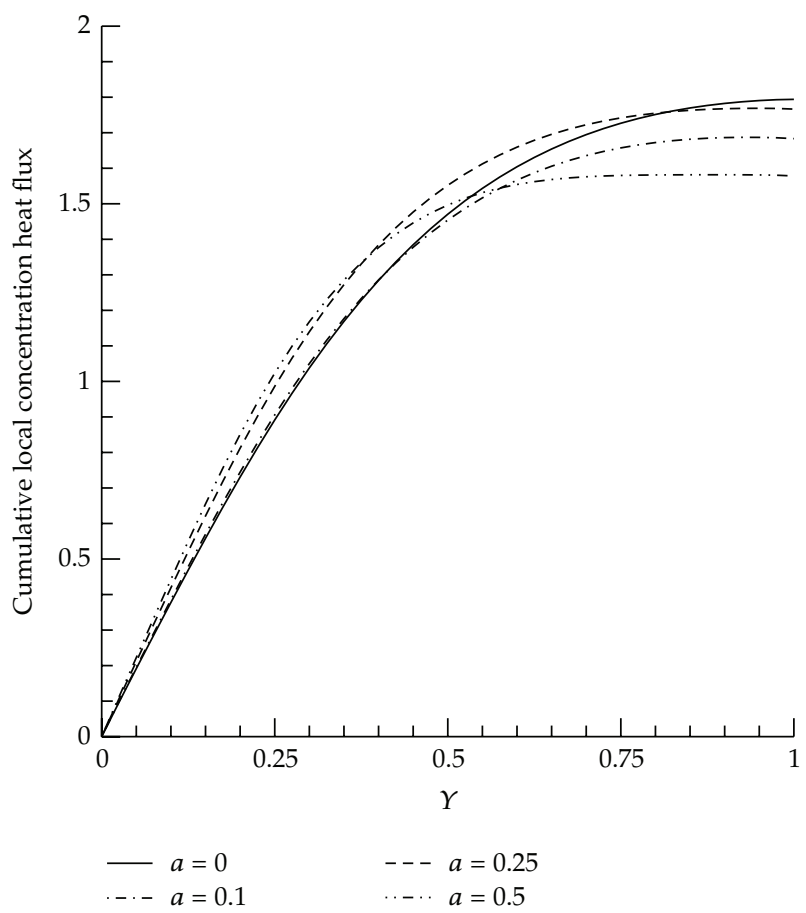

Figure 7: Cumulative mass flux plots along the left vertical wall while fixing $\operatorname{Le}=1, B=1,(D / H)=0.1$, $\mathrm{Ra}=100, S_{C}=0.1$ for different values of " $a$ ".

In Figure 7 the cumulative mass fluxes (CMFLXs) along the left vertical wall are presented. At all values of " $a$ " similar to CHFLXs, the CMFLXs increase along the left vertical wall. With increasing " $a$ " along the leading part of the left vertical wall a raise in CMFLXs are noticed. However, owing to the presence of concentration stratification, the extent of the stretch along the left vertical wall wherein such a raise is noticed decreases from $0 \leq Y<0.5$ to $0 \leq Y<0.4$.

Again owing to the presence of concentration stratification, the variation in CMFLXs along the upper portion of the left vertical wall (i.e., $0.5<Y \leq 1$ ) gets complex. An initial raise in " $a$ ", 0.0 to 0.1 leads to a fall in CMFLXs, corresponding to $0.5<Y \leq 1$, and thereby a fall in global cumulative mass flux (GCMFLX) or Sherwood number (Sh). A further increase in " $a$ " from 0.1 to 0.25 leads to shoot-up in CMFLXs and GCMFLX and on further increasing " $a$ " to 0.5 a significant fall in CMFLXs and GCMFLX is noticed. To understand such a trend in mass fluxes the concentration distribution in the whole domain for different values of " $a$ " is traced and presented in Figures 8(a)-8(d).

The isoconcentration contour pattern depicted by plots clearly indicates the additional dimension to the complexity of interaction between various forces responsible for concentration transport in the porous enclosure. One can notice the presence of concentration boundary layers, whose characteristics are sensitive to $I / S$ velocity, along the left vertical wall. The plots clearly depict an overall increase in the concentration transport with increasing values of " $a$ " that is, $I / S$ velocity. With the increase in " $a$ " circular packets of concentric concentration are seen to manifest on the $I / S$ windows. 




(a)

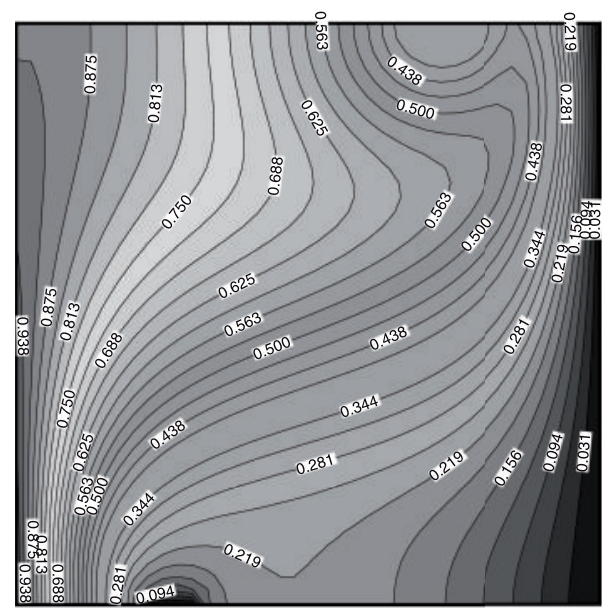

(c)

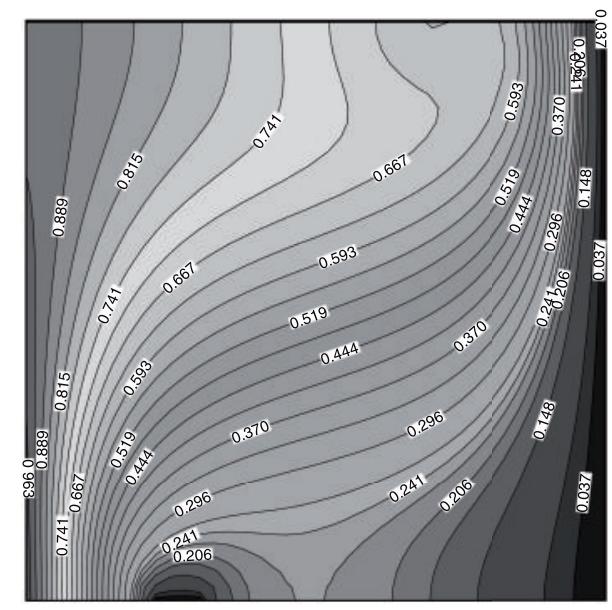

(b)



(d)

Figure 8: Isoconcentration contours along the left vertical wall while fixing $\operatorname{Le}=1, B=1,(D / H)=0.1$, $\mathrm{Ra}=100, S_{C}=0.1$ for (a) $a=0.0$, (b) $a=0.1$, (c) $a=0.25$, (d) $a=0.5$.

\subsection{Influence of $I / S$ Window Width $(D / H)$}

The influence of size of $I / S$ window on the flow, temperature, and concentration fields is analyzed and presented in Figures 9(a) and 9(b) and Figures 10(a)-10(i). In Figures 9(a) and 9(b) CHFLX and CMFLX along the left vertical wall are presented for $0 \leq(D / H) \leq 0.3$, Le $=1, B=1, \mathrm{Ra}=100, a=0.1, S_{C}=0.1$. At all values of $D / H$ both CHFLX and CMFLX increase as one gradually moves along the vertical wall. All along the left vertical wall both local heat and mass fluxes increase with increasing values of $D / H$. Both Nusselt number and Sherwood number are seen to increase with increasing values of $D / H$. In Figures 10(a)10(i) streamlines, isotherms, isoconcentration contours corresponding to $(D / H)=0.1,0.2$, $0.3, B=1, \mathrm{Le}=1, \mathrm{Ra}=100$ and $a=0.1, S_{C}=0.1$. From the streamlines in Figures 10 (a) -10 (c) one can notice the manifestation of a prominent primary circulation zone in the core of the domain and two secondary circulations centered on the $I / S$ window slits at all 


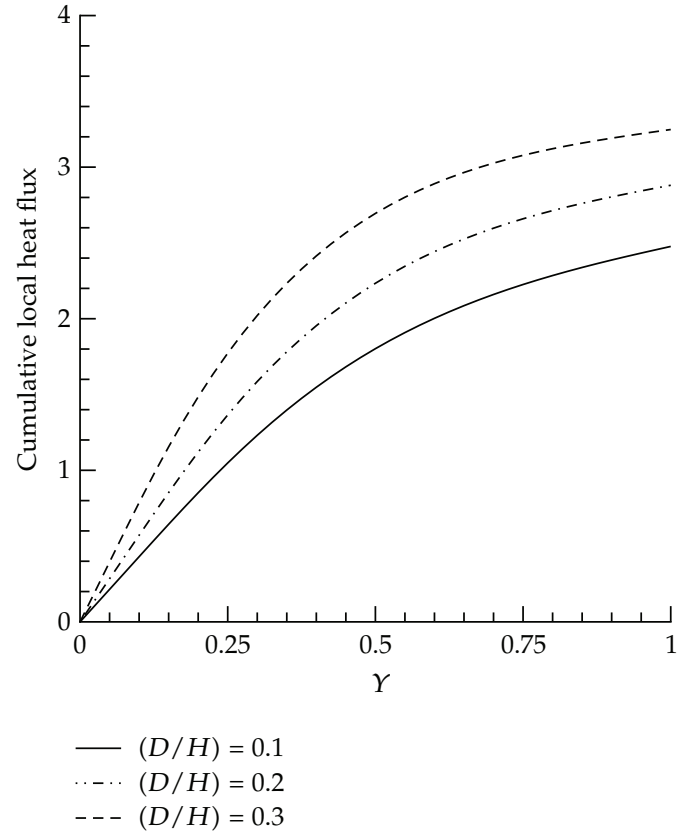

(a)

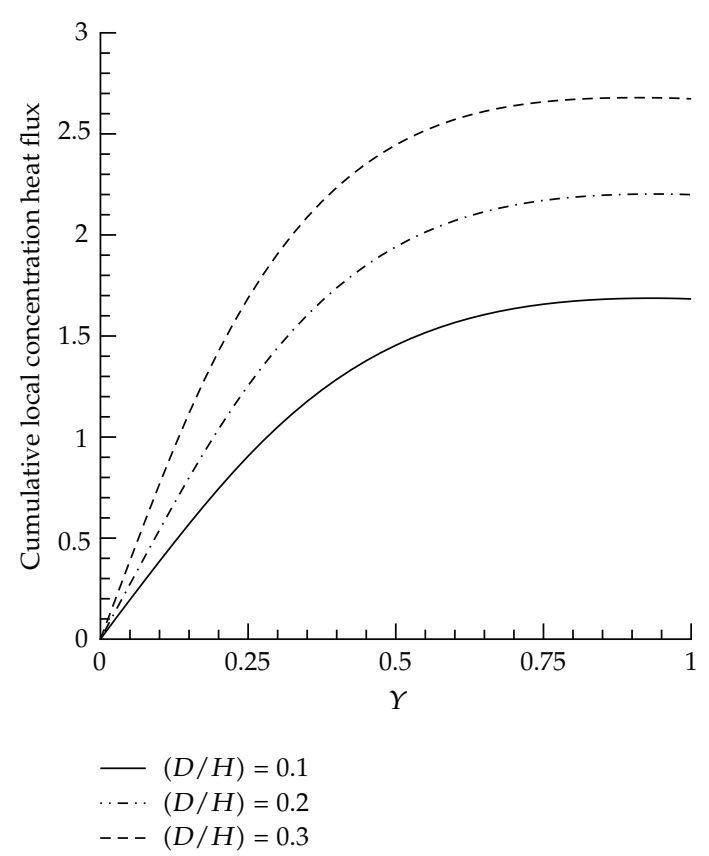

(b)

Figure 9: (a) Cumulative heat flux and (b) cumulative mass flux plots along the left vertical wall while fixing $\mathrm{Le}=1, B=1, a=0.1, \mathrm{Ra}=100, S_{C}=0.1$ for different values of $D / H$.

values of $D / H$. Increasing the $I / S$ window slit size leads to overall enlargement of both the primary and secondary circulation zones. From the isotherm plots in Figures 10(d)-10(f) one can notice the presence of TBLs along the left vertical walls. These TBLs get sharper with increasing values of $D / H$. The change in the isotherm pattern and magnitudes of isotherms suggests an overall enhancement in heat transfer in the enclosure with increasing $D / H$. From Figures 10(g)-10(i) similar variation is noticed in concentration fields, with the increase in $D / H$. Concentration boundary layers (CBLs) noticed along the left vertical wall get sharper with increasing values of $D / H$.

\subsection{Influence of Buoyancy Ratio " $B$ "}

To analyze the influence of buoyancy ratio " $B$ ", which denotes the relative strengths of two buoyancy forces, namely, mass and thermal buoyancy forces, simulations are carried out for a wide range of " $B$ ", covering $-1.5 \leq B \leq 1.5$. Here it can be noted that the algebraic sign of " $B$ " denotes the effective direction of these buoyancy forces. Thermal buoyancy forces act vertically upward, and the species buoyancy may act in either direction depending on the molecular weight relative to the fluid. A heavier species contributes to a buoyant force that acts vertically downward, thereby opposing the vertically upward thermal buoyancy, and is negative in sign. If $B=0$, the flow is driven by buoyancy alone. Simulations have been carried out with different degrees of mass stratifications. In Figure 11(a), CHFLXs along the left vertical wall are plotted for different values of " $B$ " when Le $=1, D / H=0.1, a=0.1$, 


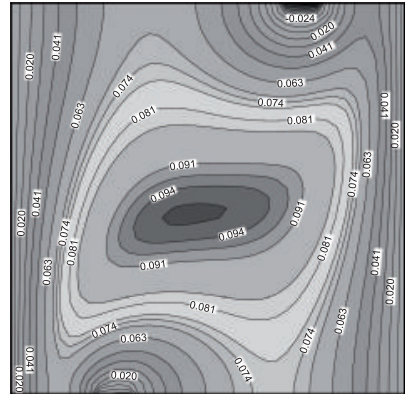

(a)



(d)

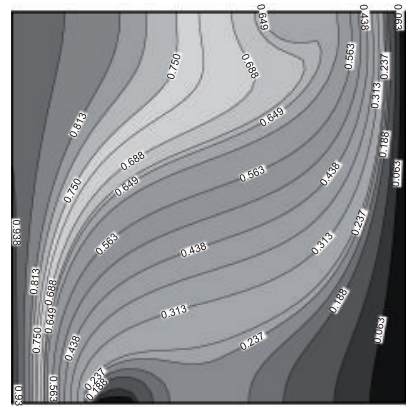

(g)

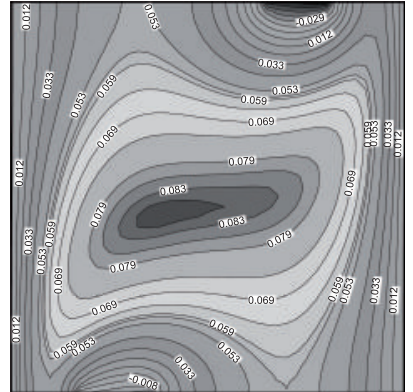

(b)



(e)

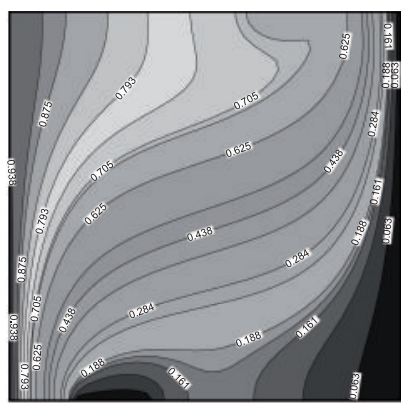

(h)



(c)



(f)

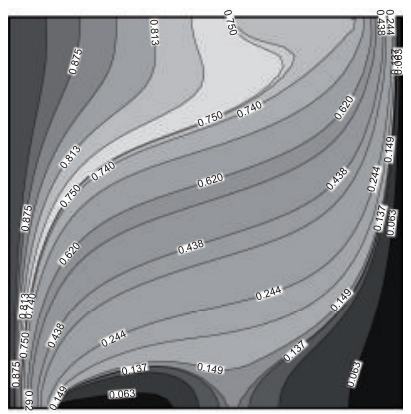

(i)

Figure 10: Streamline contours along the left vertical wall while fixing Le $=1, B=1, a=0.1, \mathrm{Ra}=100$, $S_{C}=0.1$ for streamline plots $(\mathrm{a})(D / H)=0.1$, (b) $(D / H)=0.2$, (c) $(D / H)=0.3$. Corresponding isotherm and isoconcentration contours are in $(\mathrm{d}-\mathrm{f})$ and $(\mathrm{g}-\mathrm{i})$, respectively.

$\mathrm{Ra}=100, S_{C}=0.1$. From Figure 11(a) one can see that CHFLXs increase with increasing values of " $B$ ". All along the left vertical wall local heat fluxes (LHFLXs) are in a raise as " $B$ " is raised. At all values of " $B$ " a sharp raise in CHFLXs is noticed along the leading edge of the left vertical wall, that is, $0 \leq Y \leq 0.4$. To understand this behavior, flow and temperature fields are traced and presented in Figure 12.

The streamlines in Figures 12(a)-12(g) clearly depict that the flow field is highly sensitive both to the sign and magnitude of " $B$ ". When the downward oriented species buoyancy forces are dominantly opposing the upward oriented thermal buoyancy forces (i.e., when $B=$ $-1.5)$, the flow is primarily covered by the elongated semicircular pattern, emanating from 


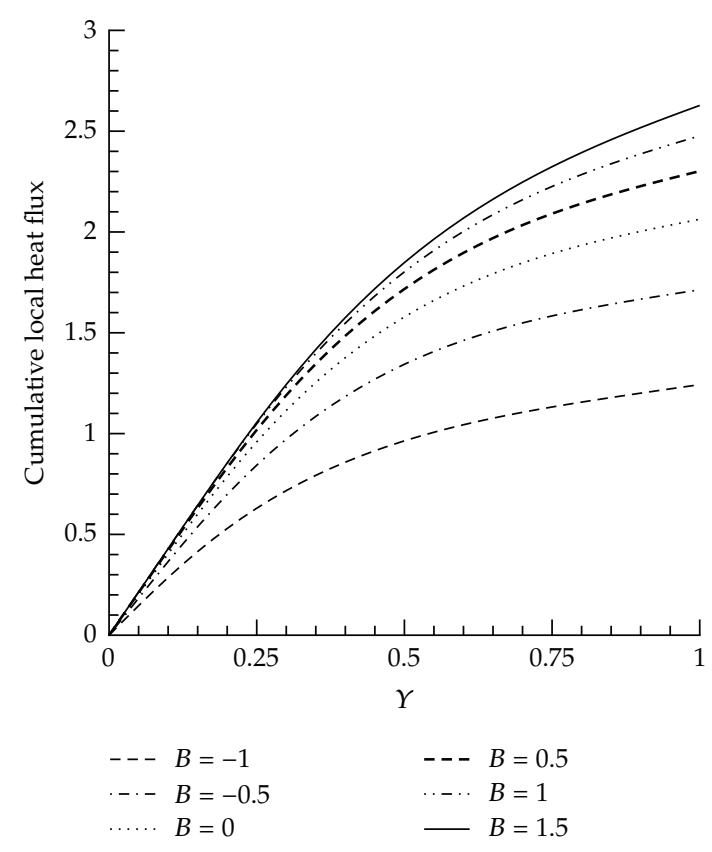

(a)

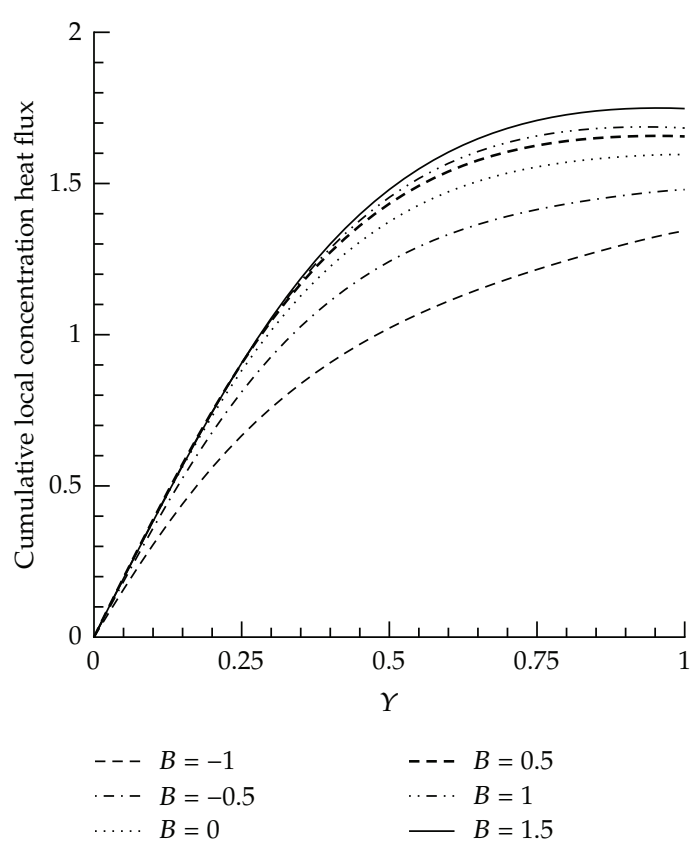

(b)

Figure 11: (a) Cumulative heat flux and (b) cumulative mass flux plots along the left vertical wall while fixing $\mathrm{Le}=1, D / H=0.1, a=0.1, \mathrm{Ra}=100, S_{C}=0.1$ for different values of " $B$ ".

the $I / S$ window located on the top horizontal wall, between two vertical flow fields which are adjacent to the vertical walls of the enclosure. As the " $B$ " is raised to -1 , where in the downward facing species buoyancy forces are opposing but are equal to the upward facing thermal buoyancy forces, the semicircular flow fields manifest on $I / S$ windows located on bottom horizontal wall and thereby reduce the dominance of downward protruding semicircular pattern that emanates from top $I / S$ window slit. A further increase in " $B$ " gradually leads to the dominance of the thermal buoyancy forces. As thermal buoyancy forces get dominant, a primary circular zone manifests between the two semicircular patterns centered on the $I / S$ window slits. With a further raise in " $B$ " to 0.5 , where in both the buoyancy forces are upward oriented, the primary circulation zone prominently falls major part of the enclosure, except the region close to the $I / S$ window slits. As the " $B$ " is raised to 1.5 one can notice that semicircular flow patterns again tend to get severe and the primary circulation zone begins to shrink in size. With a further increase in " $B$ ", the primary circulation zone completely vanishes allowing the two semicircular patterns to elongate and cover whole of the enclosure.

The corresponding isotherms and isoconcentration contours are presented in Figures 13(a) $-13(\mathrm{~g})$ and Figures 14(a)-14(g). The majority of isotherms which are vertically oriented when $B=-1.5$ gradually undergo a clockwise diagonal twist as " $B$ " is raised to -1.0 . When " $B$ " is raised to -0.5 , the diagonally oriented isotherms which emanate from the lower left corner begin to turn towards the horizontal walls, especially while they are being traced in the core of the domain. On increasing " $B$ " in steps to 1.5 while the isotherms close to the vertical wall tend to get vertical, they take a horizontal path with increasingly sharp bends 


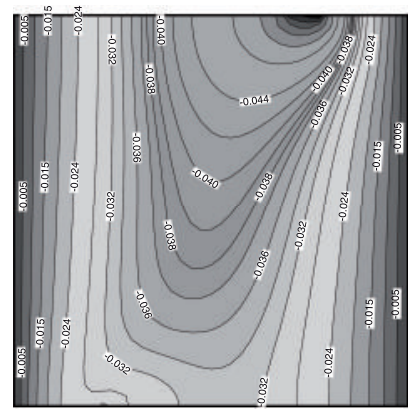

(a)



(d)

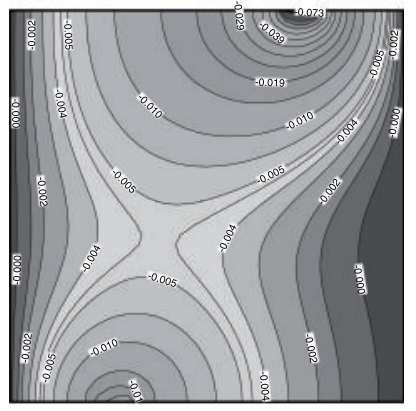

(b)

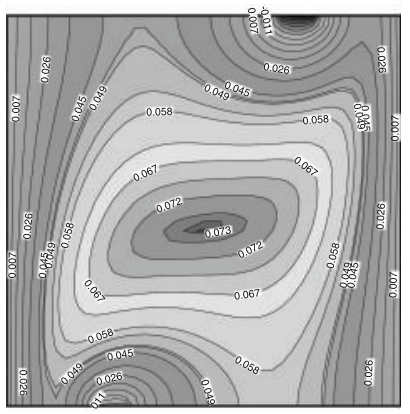

(e)

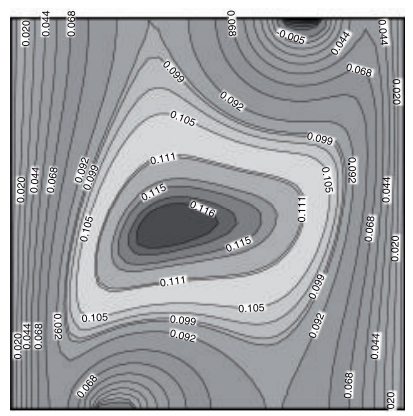

$(\mathrm{g})$

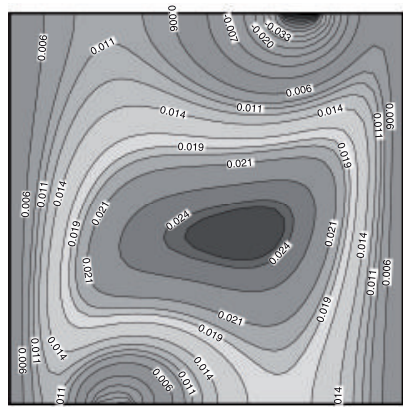

(c)

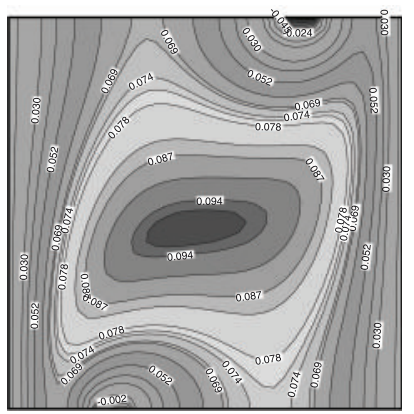

(f)

Figure 12: Streamline contours along the left vertical wall while fixing Le $=1, D / H=0.1,(a)=0.1$, $\mathrm{Ra}=100, S_{C}=0.1$ for (a) $B=-1.5$, (b) $B=-1$, (c) $B=-0.5$, (d) $B=0$, (e) $B=0.5$, (f) $B=1$, (g) $B=1.5$.

near the vertical walls. The raise in the sharpness of TBLS clearly supports the observed increase in $\mathrm{Nu}$ with increasing values of " $B$ ".

Features observed in the isoconcentration contours are nearly similar to those observed in isotherm plots especially when $-1.5 \leq B \leq 0$. The deviation in the iso-concentration contours from the corresponding isotherms when $0 \leq B \leq 1.5$ can be attributed to the presence of species stratification in the porous enclosure. Like in isotherm plots, here too one can notice the manifestation of CBLs along the vertical walls. The increase in Sherwood numbers with increasing " $B$ " (Figure $11(\mathrm{~b})$ ) is clearly supported by the increasingly sharpening CBLs with increasing " $B$ ". Both the isotherm and isoconcentration patterns depict an enhanced heat and species transportation in the enclosure with increasing values of " $B$ ". 


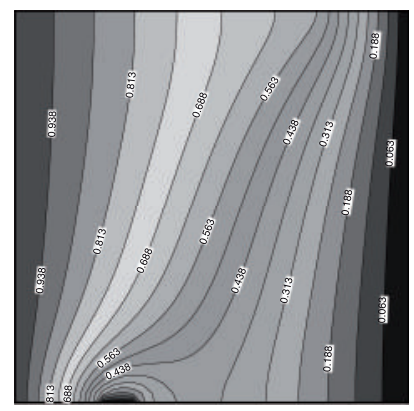

(a)

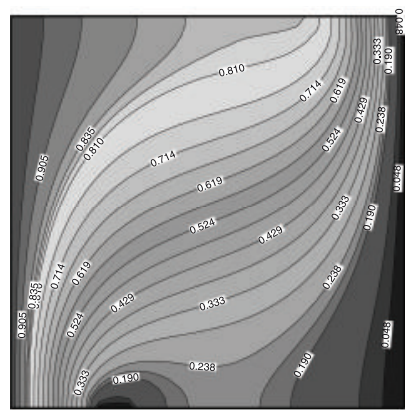

(d)

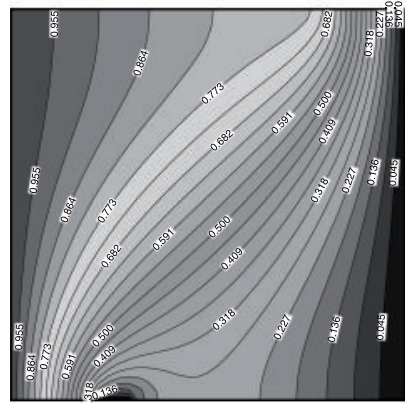

(b)



(e)



(g)

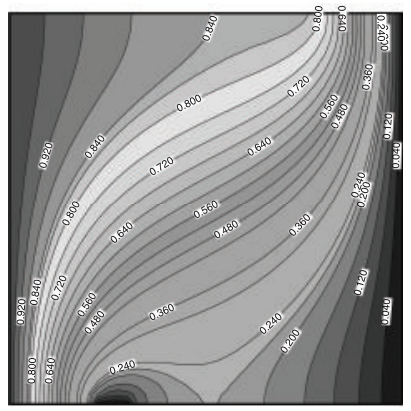

(c)



(f)

Fure 13: Isotherm contours along the left vertical wall while fixing $\mathrm{Le}=1, D / H=0.1,(a)=0.1, \mathrm{Ra}=100$ $S_{C}=0.1$ for (a) $B=-1.5$, (b) $B=-1$, (c) $B=-0.5$, (d) $B=0$, (e) $B=0.5$, (f) $B=1$, (g) $B=1.5$.

\subsection{Influence of Lewis Number "Le"}

In Figure 15(a) influence of Le on CHFLX along the left vertical wall is presented. Along the leading edge of the left vertical wall (i.e., for $0 \leq Y \leq 0.25$ ) CHFLXs remain insensitive to the differences in thermal and mass diffusivities. However along $0.25 \leq Y \leq 1$ one can notice a fall in CHFLX all along the wall with increasing values of Le. Consequently GCHFLX or Nu decreases with increasing "Le". It is also to be noticed that at all values of Le, there is a gradual increase in CHFLX as one traverses along the left vertical wall (i.e., $0 \leq Y \leq 1$ ). When the mass diffusivity is larger than thermal diffusivity, the corresponding heat fluxes and $\mathrm{Nu}$ are larger. This indicates the possibility of greater mixing of hot and cold fluids with increasing "Le" and thereby leading to an overall raise in the temperature in the porous enclosure. To 


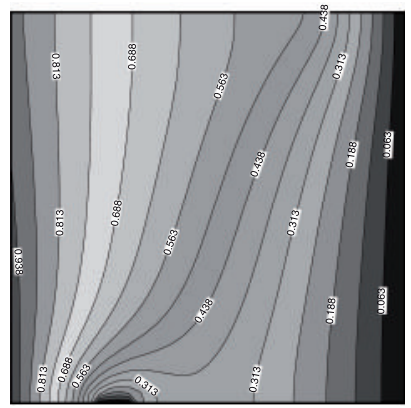

(a)



(d)

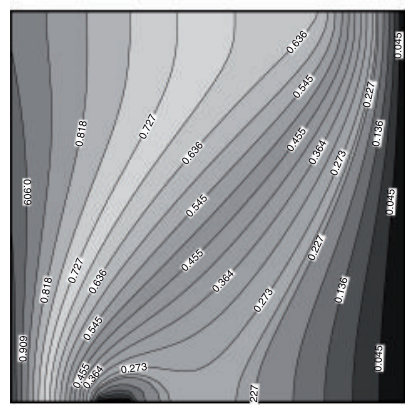

(b)

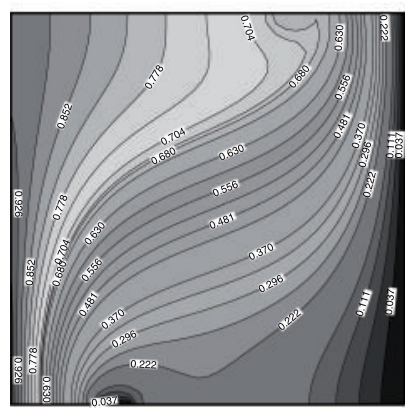

(e)

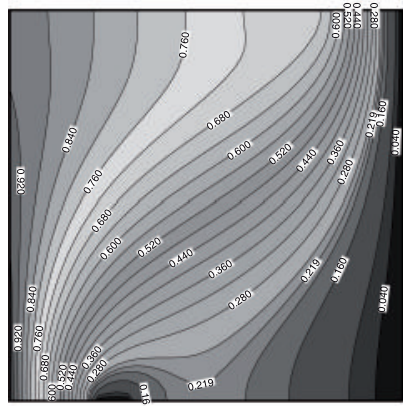

(c)

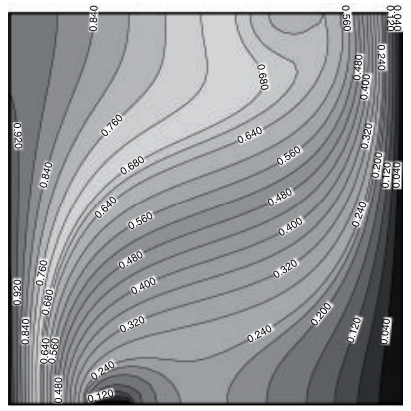

(f)

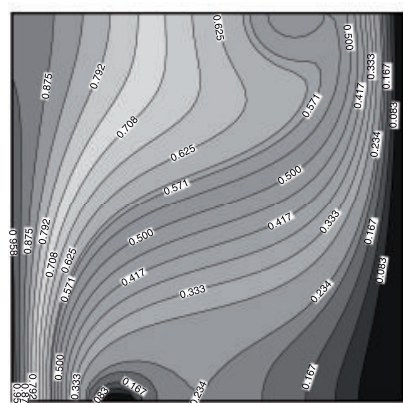

$(\mathrm{g})$

Figure 14: Isoconcentration contours along the left vertical wall while fixing Le $=1, D / H=0.1,(a)=0.1$, $\mathrm{Ra}=100, S_{C}=0.1$ for (a) $B=-1.5$, (b) $B=-1$, (c) $B=-0.5$, (d) $B=0$, (e) $B=0.5$, (f) $B=1$, (g) $B=1.5$.

further investigate such a happening, flow and temperature fields are traced in the form of streamlines and isotherms in Figures 16 and 17.

From the streamline plots in Figures 16(a)-16(d) firstly one can notice the manifestation of prominent primary circulation centered in the core of the domain, also the presence of secondary semi-circular zones centered on the $I / S$ window slits on the horizontal walls. The increase in the diagonal stretch in the primary zone and the swelling of the secondary zones leading to the corner elongation/stretching of the primary circulation zones clearly depict the increase in the mixing of hot and cold fluids with increase in "Le". Also from the isotherm plots in Figures 17(a)-17(d) one can see the presence of TBLS along the left vertical wall. The effect of increasing thermal diffusivities is clear from the increase in the slope of isotherms, especially in the core of the domain. The semi-circular isotherm pattern 


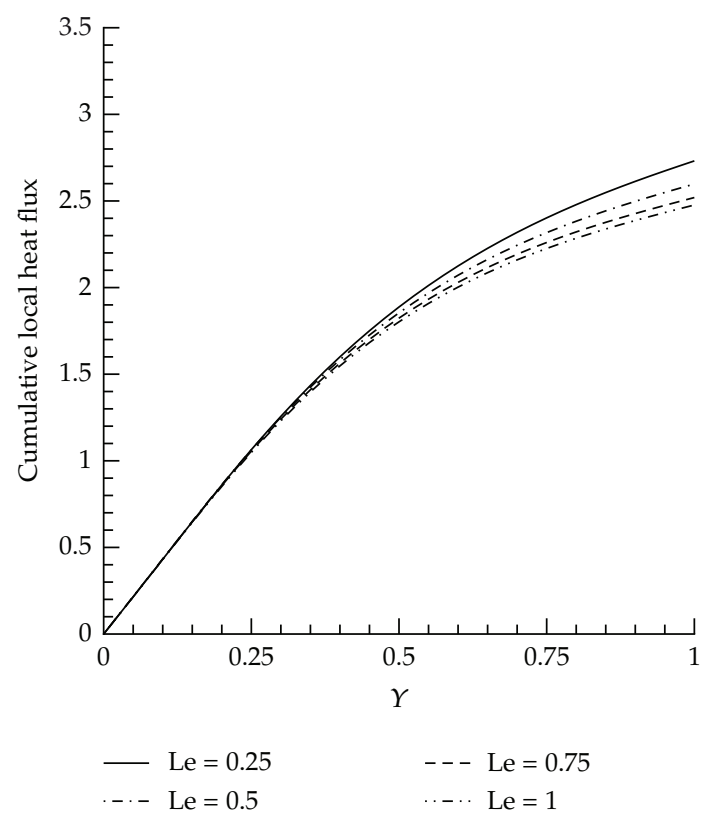

(a)

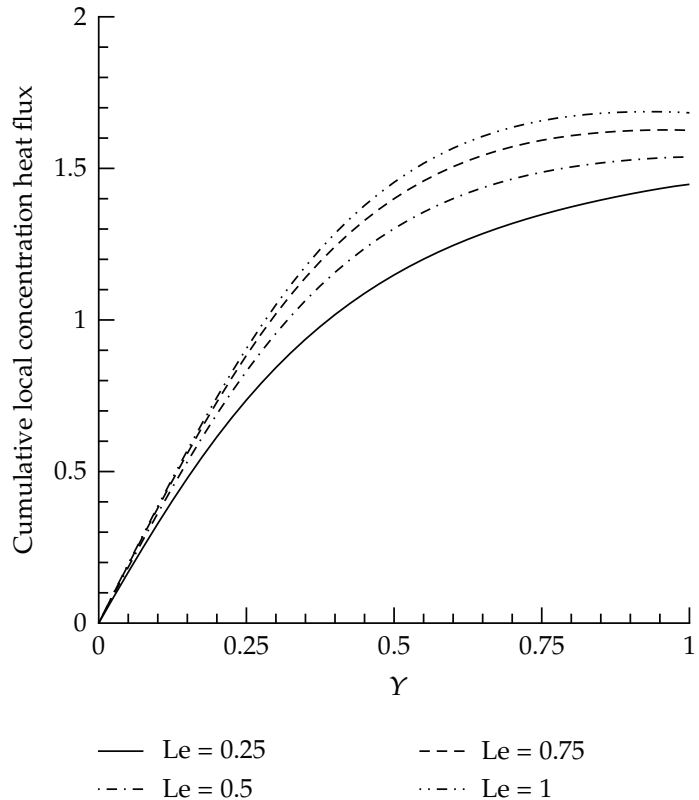

(b)

Figure 15: (a) Cumulative heat flux plots and (b) cumulative mass flux plots along the left vertical wall while fixing $B=1,(D / H)=0.1, a=0.1, \mathrm{Ra}=100, S_{C}=0.1$ for different values of "Le".

seen in the leading portion of the lower horizontal wall is due to injection of fluid at ambient temperature through the $I / S$ window. In Figure 15(b) CMFLXs, corresponding to above set of parameters, along the left vertical wall are presented for different values of "Le". All along the wall CMFLXs increase with increasing values of "Le". Consequently GCMFLX or Sherwood number (Sh) increases with increasing "Le". It is also to be noted that while the magnitudes of Sh are lower than $\mathrm{Nu}$, their relative raise with increasing "Le" is considerably larger than the relative fall in Nu. From Figure 15(b) one can also notice that there is sharp raise in CMFLXs along the leading edge of the left vertical wall, that is, $0 \leq Y \leq 0.4$, and thereby CMFLXs are seen to increase gradually. To further investigate the concentration contours are traced in Figures 18(a)-18(d). From the iso-concentration plots in Figures 18(a)18(d) one can notice the manifestation of sharp concentration boundary layer (CBL) along the left vertical wall. These CBLs tend to get blunt as one moves beyond $Y=0.4$. From the contour magnitudes one can also notice overall increase in the concentration level in the core of the enclosure with increasing "Le". Clearly increase in thermal diffusivities favors the concentration transport.

\subsection{Influence of Rayleigh Number "Ra"}

In Figure 19(a) influence of "Ra" on CHFLX along the left vertical wall is presented for $10 \leq$ $\mathrm{Ra} \leq 150, B=1,(D / H)=0.1, a=0.1, \mathrm{Le}=1, S_{C}=0.1$. Clearly CHFLX increases as one traverses the left vertical wall starting from the lower left corner. Also all along the left vertical wall LHFLXs are seen to increase with increasing values of "Ra". GHFLX or Nu increases 


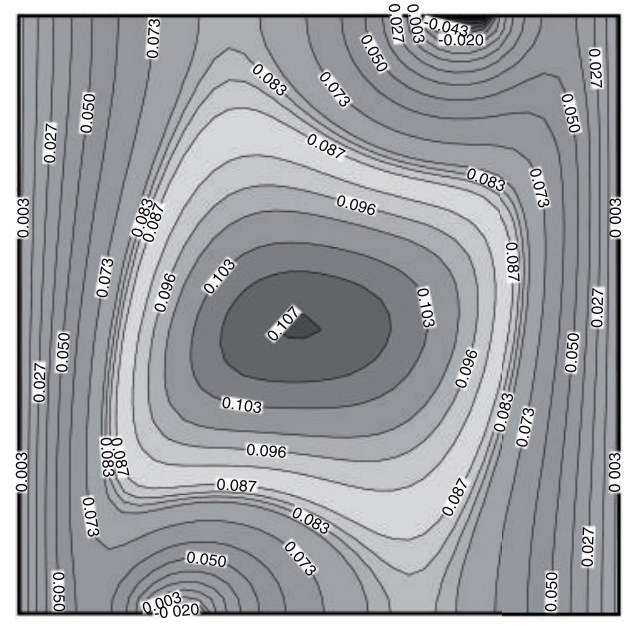

(a)

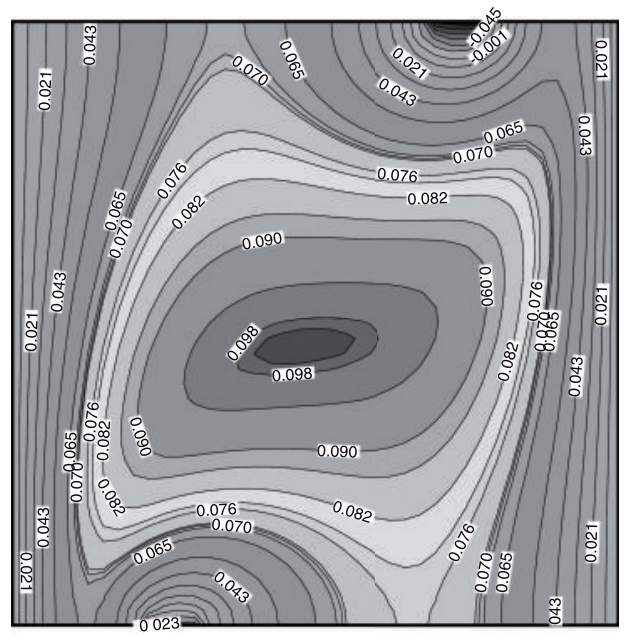

(c)

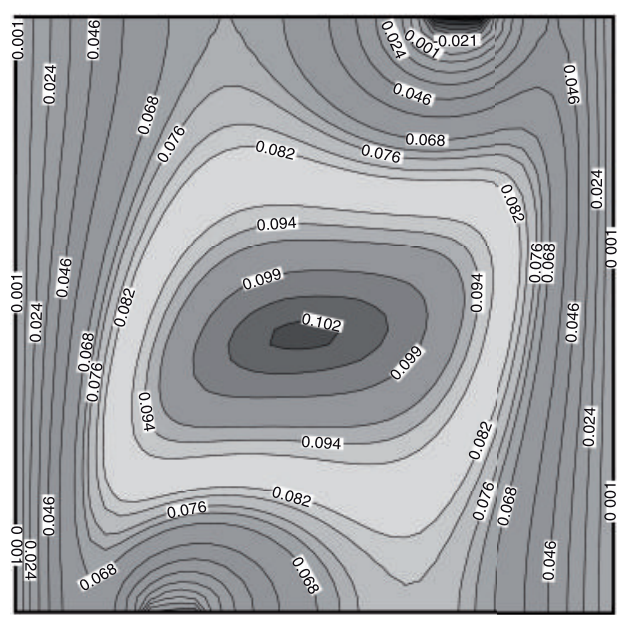

(b)

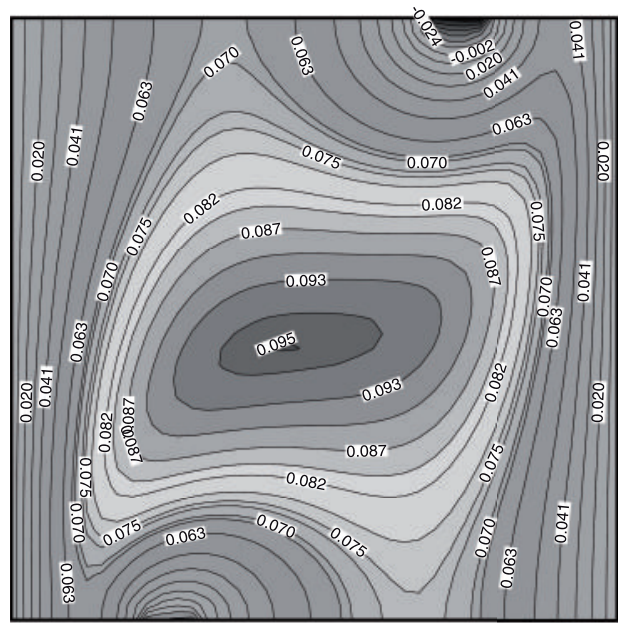

(d)

Figure 16: Streamline contours along the left vertical wall while fixing $B=1, D / H=0.1, a=0.1, \mathrm{Ra}=100$, $S_{C}=0.1$ for (a) Le $=0.25$, (b) $\mathrm{Le}=0.5$, (c) $\mathrm{Le}=0.75$, (d) $\mathrm{Le}=1.0$.

with "Ra". In Figure 19(b) CMFLXs along the left vertical wall are presented for the above set of parameters. Clearly CMFLX increases as one marches along the left vertical wall, with a relatively sharp raise when $0 \leq Y \leq 0.5$. At any point along the left vertical wall LHFLX is seen to increase with increasing "Ra". Owing to the presence of mass stratification in the porous enclosure, while the GMFLX or Sherwood number undergoes a considerable enhancement for $10 \leq \mathrm{Ra} \leq 75$, it changes marginally when $75<\mathrm{Ra}<150$. To further analyze the situation the flow, temperature, and concentration fields are analyzed as streamlines, isotherms, and iso-concentration contours in Figures 20, 21 and 22.

In Figures 20(a)-20(f) streamlines corresponding to $B=1,(D / H)=0.1, a=0.1, \mathrm{Ra}=$ $100, S_{C}=0.1,10 \leq \mathrm{Ra} \leq 150$ are provided. In the range $10 \leq \mathrm{Ra} \leq 75$ there is a considerable variation in streamline pattern as "Ra" is increasing. At all values of " $\mathrm{Ra}$ " one can notice the 


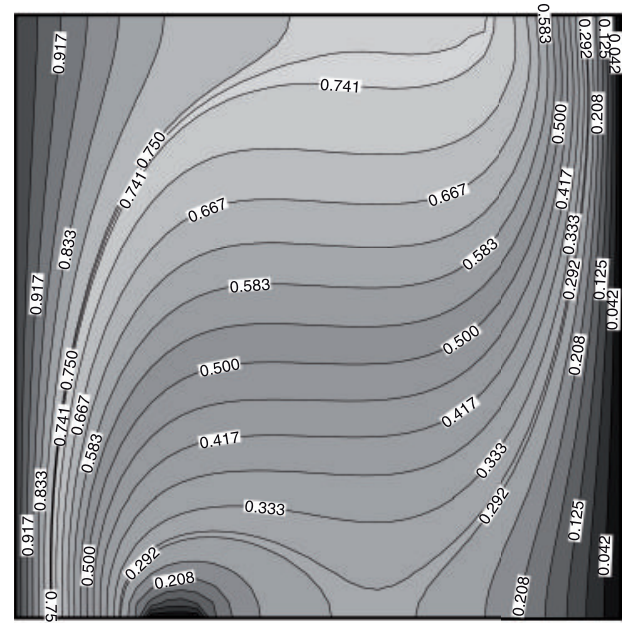

(a)



(c)

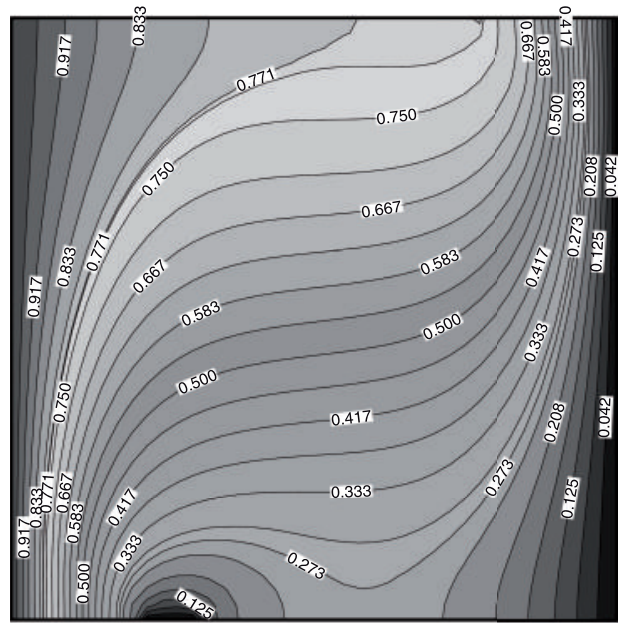

(b)

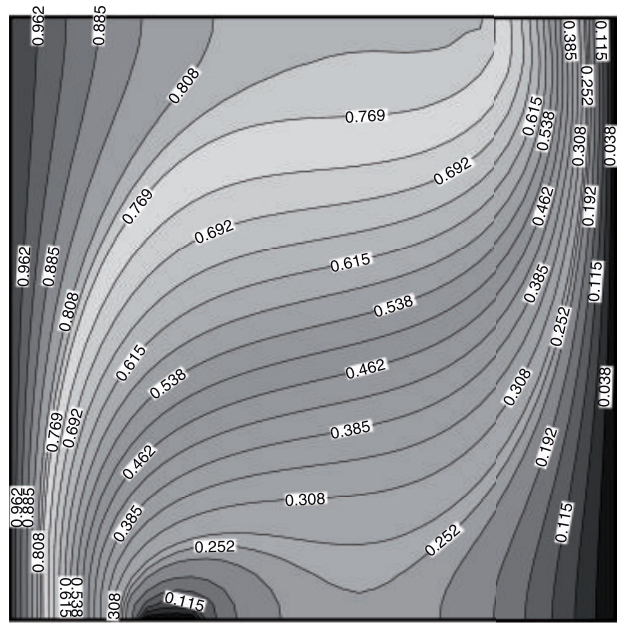

(d)

Figure 17: Isotherm contours along the left vertical wall while fixing $B=1, D / H=0.1, a=0.1, \mathrm{Ra}=100$, $S_{C}=0.1$ for (a) Le $=0.25$, (b) Le $=0.5$, (c) Le $=0.75$, (d) Le $=1.0$.

presence of both the primary circulation zone centered in the middle of the enclosure and two secondary circulation zones centered on the $I / S$ window slits. The primary circulation zone which is vertically oriented and is squeezed between the vertical flows due to injection and suction effects gradually undergoes diagonal bulging as "Ra" is increased. With the increase in "Ra", the breath of the two vertical flow patterns adjacent to the vertical wall tends to decrease. For $150 \geq \mathrm{Ra} \geq 75$ relatively a small variation is noticed in streamline pattern. The enlargement in the primary circulation for $10 \leq \mathrm{Ra}<75$ suggests a better mixing of hot and cold fluid suggesting a possibility of sustained increase in the sharpness of TBLs in corresponding isotherms.

Now in Figures 21(a)-21(f) isotherms corresponding to the above set of parameters are provided. From the isotherm pattern and the magnitude one can notice the presence of TBLs 




(a)



(c)



(b)

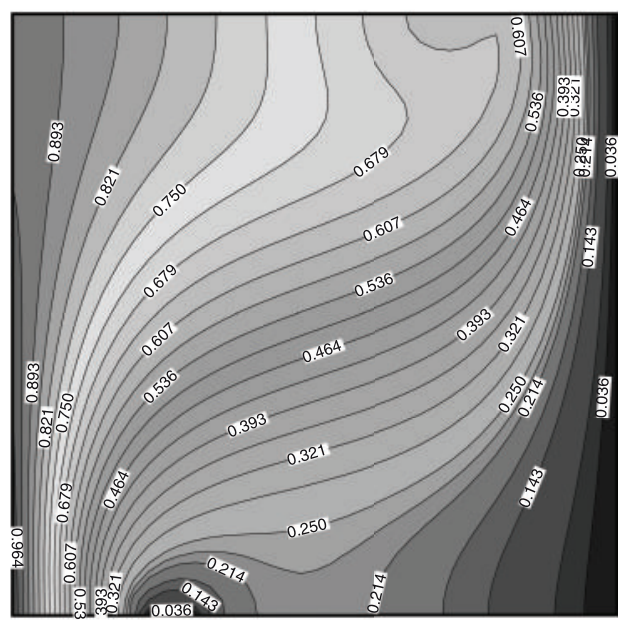

(d)

Figure 18: Isoconcentration contours along the left vertical wall while fixing $B=1, D / H=0.1, a=0.1$, $\mathrm{Ra}=100, S_{C}=0.1$ for (a) Le $=0.25$, (b) Le $=0.5$, (c) Le $=0.75$, (d) Le $=1.0$.

with increasing sharpness as "Ra" is gradually increased from 10 to 150 . On the isotherm magnitudes one can clearly notice an overall enhancement in the temperature distribution in the enclosure. In Figures 22(a)-22(f) one can notice the presence of CBLs along the left vertical wall. While the CBLs corresponding $10 \leq \mathrm{Ra} \leq 75$ get sharpened, those corresponding to Ra $>75$ undergo only a little variation especially when $Y>0.85$, that is, towards at the end of the left vertical wall. This can be attributed to the presence of concentration stratification in the enclosure. The overall concentration distribution in the enclosure is on the raise with increasing "Ra".

\section{Conclusions}

Numerical computations have been carried out on Darcian double diffusive mixed convection process in a in a fluid saturated porous enclosure under $I / S$ effect with mass 


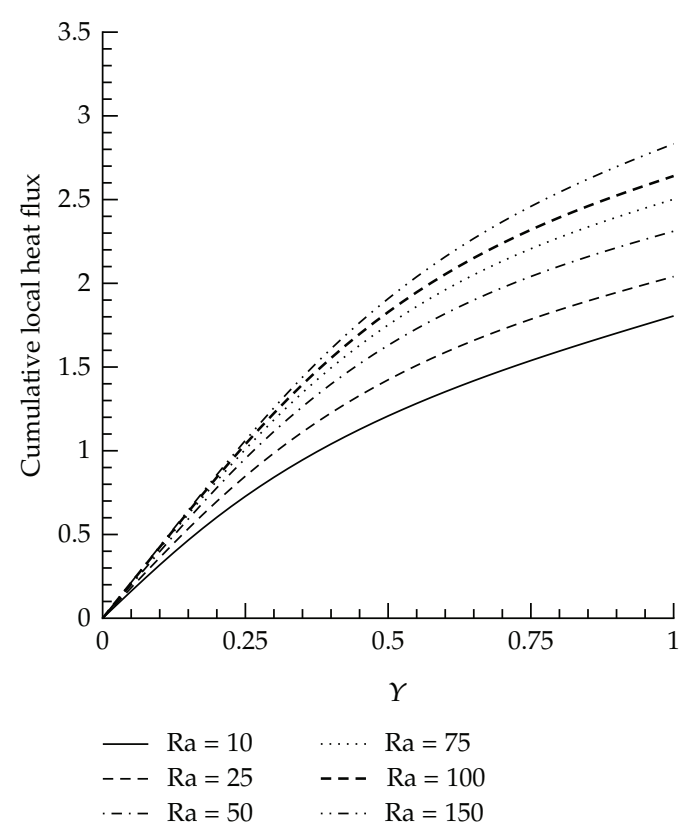

(a)

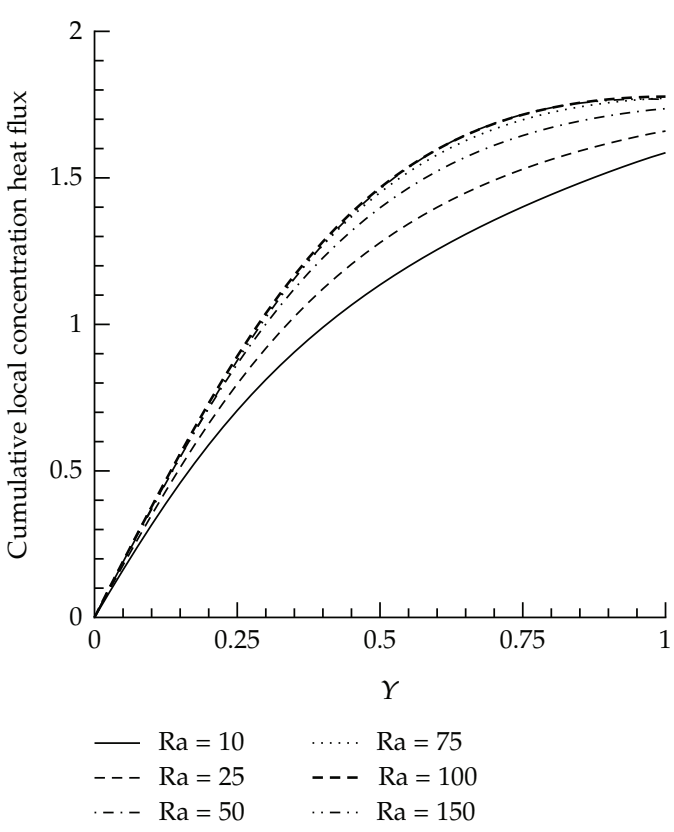

(b)

Figure 19: (a) Cumulative heat flux plots and (b) cumulative mass flux plots along the left vertical wall while fixing $B=1,(D / H)=0.1, a=0.1$, Le $=1, S_{C}=0.1$ for different values of "Ra".

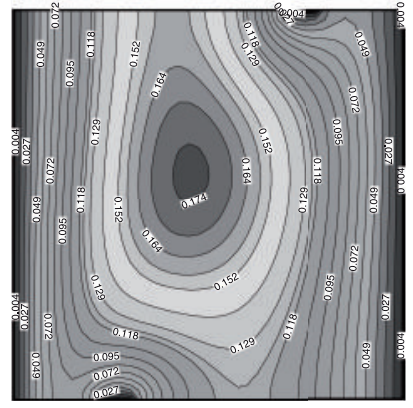

(a)

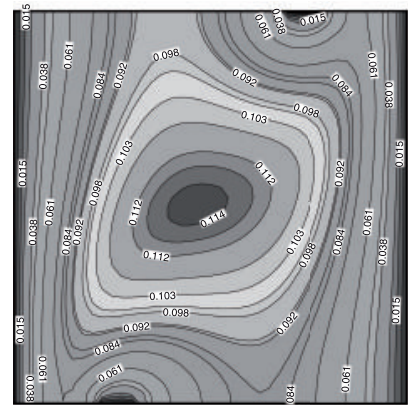

(d)

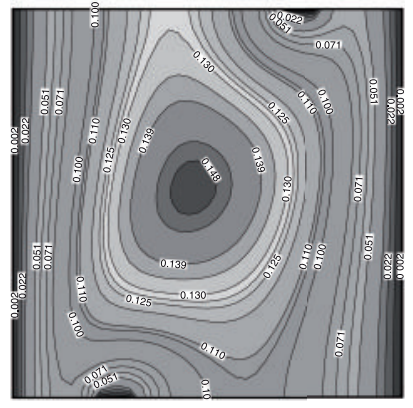

(b)

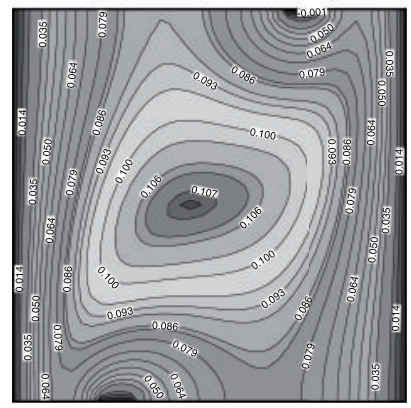

(e)

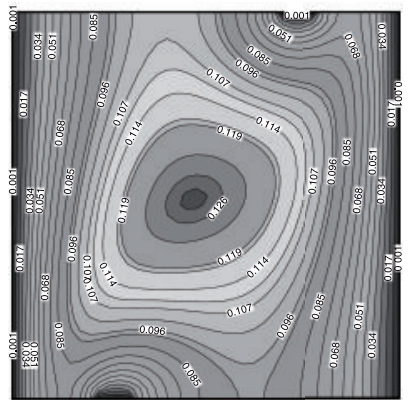

(c)



(f)

Figure 20: Streamline contours along the left vertical wall while fixing $B=1,(D / H)=0.1, a=0.1$, $\mathrm{Ra}=100, S_{C}=0.1$ for (a) $\mathrm{Ra}=10$, (b) $\mathrm{Ra}=25$, (c) $\mathrm{Ra}=50$, (d) $\mathrm{Ra}=75$, (e) $\mathrm{Ra}=100$, (f) $\mathrm{Ra}=150$. 


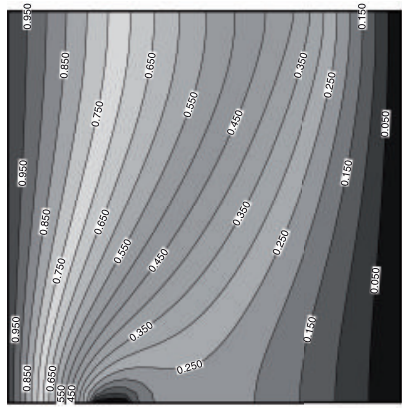

(a)

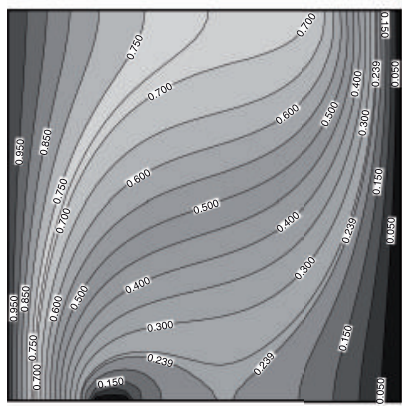

(d)



(b)

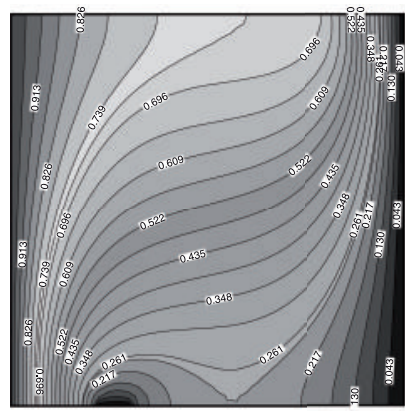

(e)

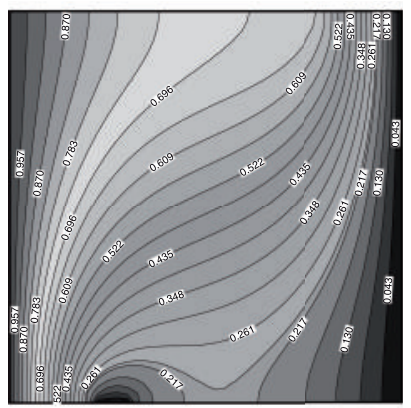

(c)

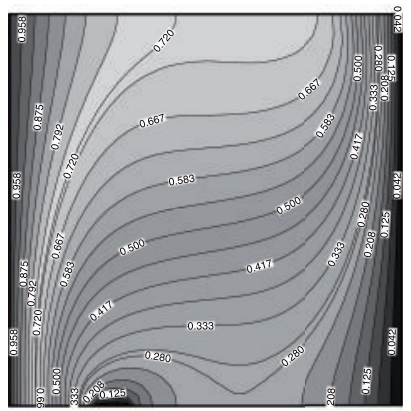

(f)

Figure 21: Isotherm contours along the left vertical wall while fixing $B=1,(D / H)=0.1, a=0.1, \mathrm{Ra}=100$, $S_{C}=0.1$ for (a) $R a=10$, (b) $R a=25$, (c) $R a=50$, (d) $R a=75$, (e) $R a=100$, (f) $R a=150$.

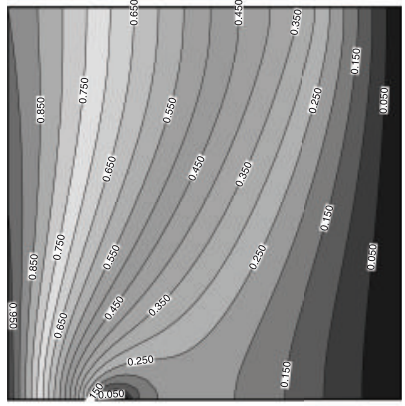

(a)

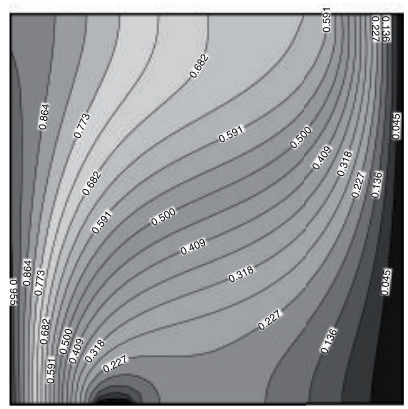

(d)

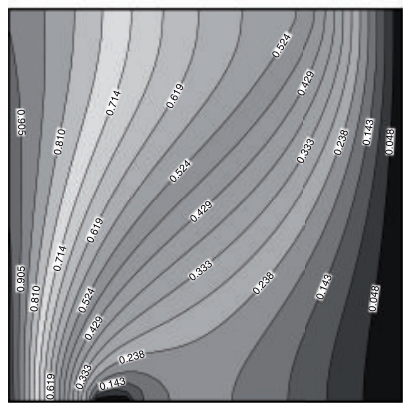

(b)

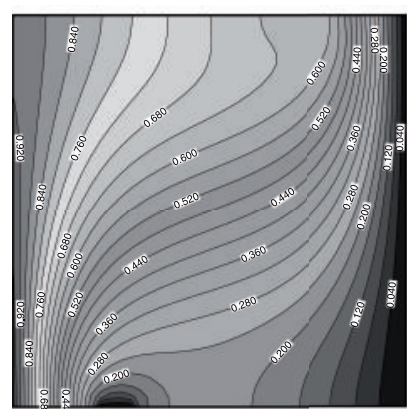

(e)



(c)

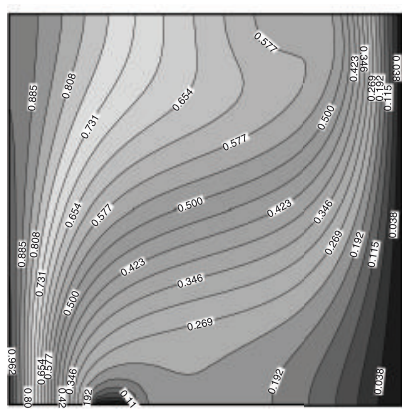

(f)

Figure 22: Isoconcentration contours along the left vertical wall while fixing $B=1,(D / H)=0.1, a=0.1$, $R a=100, S_{C}=0.1$ for (a) $R a=10$, (b) $R a=25$, (c) $R a=50$, (d) $R a=75$, (e) $R a=100$, (f) $R a=150$. 
stratification by finite element method. Flow, temperature, and concentration fields are analyzed through streamline, isotherm, iso-concentration tracing and local and cumulative heat flux, cumulative concentration heat flux calculations. Following are the conclusions based on the results obtained from the present study.

(i) While $\mathrm{Nu}$ decreases with increasing $I / S$ velocity, Sh is found to initially decrease but later increase. Prominent primary circulation pattern with secondary circulation zones that stretch on increasing $I / S$ velocity manifests in flow field. TBLs and CBLs, thermal and mass gradients highly sensitive to the $I / S$ velocity are noticed in the temperature and concentration fields.

(ii) Both $\mathrm{Nu}$ and $\mathrm{Sh}$ increase with increasing size of $I / S$ window $(D / H)$. Increasing $(D / H)$ enlarges both primary and secondary circulation zones and sharpens the TBLs and CBLs.

(iii) Flow pattern undergoes significant pattern variation as the opposing thermal and mass buoyancy forces turn to favor each other. Semicircular flow pattern is transformed to a multicellular flow with a prominent primary circulation zone. Both $\mathrm{Nu}$ and $\mathrm{Sh}$ increase with increasing values of " $B$ ".

(iv) While $\mathrm{Nu}$ decreases with increasing Le, it increases with increasing "Ra". Sh is found to increase both with increasing Le and Sh. Flow, temperature, and concentration fields are sensitive both to "Le" and "Ra".

(v) Darcy case of an increase in the levels of concentration stratification leads to a fall in $\mathrm{Nu}$ and $\mathrm{Sh}$ values.

\section{Nomenclature}

a: $\quad$ Suction/injection velocity $\left(=V_{0} / V_{c}\right)$

B: $\quad$ Buoyancy ratio

c: Dimensionless species concentration

C: Nondimensional species concentration

d: $\quad$ Mass diffusivity

$D$ : Width of the inlet

$D / H$ : Dimensionless width of the inlet

$e: \quad$ Typical element in finite element formulation

g: Gravitational acceleration

$H$ : Height of the cavity

$k$ : Thermal conductivity

$K$ : $\quad$ Permeability of the porous medium

$n$ : $\quad$ Outward unit normal to the surface

$N_{i}$ : Quadratic interpolation function

$\mathrm{Nu}$ : Nusselt number

Ra: $\quad$ Rayleigh number $\left(=K g \beta H\left(T_{w}-T_{0}\right) / \alpha v\right)$

$s_{c}: \quad$ Dimensional mass stratification parameter

$S_{c}$ : Nondimensional mass stratification parameter

$T$ : $\quad$ Nondimensional temperature

$t_{0}$ : Temperature of the flow through at the inlet

$t_{w}$ : Temperature of the left vertical wall

$u, v$ : Dimensional velocity components in $x$ and $y$ directions 
$U, V: \quad$ Nondimensional velocity components in $X$ and $Y$ directions $\left(U=u / V_{c}, V=v / V_{c}\right)$

$V_{c}: \quad$ Convective velocity $\left(=g \beta K\left(T_{w}-T_{0}\right) / v\right)$

$V_{0}$ : Absolute value of the velocity of the forced flow at the inlet

$W: \quad$ Galerkin weight function used in the finite element formulation

$x, y: \quad$ Dimensional cartesian coordinates

$X, Y$ : Nondimensional cartesian coordinates $(X=x / H, Y=y / H)$

$\left(X_{1}, 0\right)$ : Coordinates of the suction/injection starting point on the bottom wall

$\left(X_{2}, 1\right)$ : Coordinates of the suction/injection starting point on the top wall.

\title{
Greek Symbols
}

$\alpha$ : Thermal diffusivity

$\beta_{t}:$ Thermal expansion coefficient $\left(=-(1 / \rho)(\partial \rho / \partial t)_{P, \mathrm{c}}\right)$

$\beta_{c}$ : Concentration expansion coefficient $\left(=-(1 / \rho)(\partial \rho / \partial c)_{P, t}\right)$

$\Gamma$ : Boundary of the domain

$\Psi$ : Nondimensional stream function

$v$ : Kinematic viscosity of fluid

$\Omega$ : Domain considered in the problem

$\rho$ : Fluid density $\left(=\rho_{0}\left[1-\beta\left(T-T_{0}\right)\right]\right.$

$\theta$ : Dimensionless temperature.

\section{Subscripts}

\author{
$0, \infty$ : Ambient points \\ $P$ : $\quad$ Pressure \\ $w$ : Evaluated at wall temperature \\ $x$ : $\quad$ Evaluated at point $x$.
}

\section{References}

[1] D. A. Nield and A. Bejan, Convection in Porous Media, Springer Verlag, New York, NY, USA, 1999.

[2] D. Ingham and I. Pop, Transport Phenomena in Porous Media, Elsevier, Oxford, UK, 2005.

[3] K. Vafai, Hand Book of Porous Media, Taylor \& Francis, New York, NY, USA, 2nd edition, 2005.

[4] I. Pop and D. Ingham, Convective Heat Tranfer: Mathematical and Computational Modelling of Viscous Fluid and Porous Media, Pergamon, Oxford, UK, 2001.

[5] A. Bejan and A. D. Krus, Heat Transfer Hand Book, Wiley, New York, NY, USA, 2003.

[6] D. Ingham, A. Bejan, and A. Mamut, Emerging Technologies and Techniques in Porous Media, Kluwer, Dordrecht, The Netherlands, 2004.

[7] D. Angirasa, "Mixed convection in a vented enclosure with an isothermal vertical surface," Fluid Dynamics Research, vol. 26, no. 4, pp. 219-233, 2000.

[8] V. J. Bansod and R. K. Jadhav, "Effect of double stratification on mixed convection heat and mass transfer from a vertical surface in a fluid-saturated porous medium," Heat TransferAsian Research, vol. 39, no. 6, pp. 378-395, 2010.

[9] A. J. Chamkha, "Non-Darcy fully developed mixed convection in a porous medium chanel with heat generation/absorption and hydro magnetic effects," Numerical Heat Transfer A, vol. 32, no. 6, pp. 653675, 1997.

[10] A. J. Chamkha, "Mixed convection flow along a vertical permeable plate embedded in a porous medium in the presence of a transverse magnetic field," Numerical Heat Transfer A, vol. 34, no. 1, pp. 93-103, 1998.

[11] W. J. Chang and W. L. Chang, "Mixed convection in a vertical tube partially filled with porous medium," Numerical Heat Transfer A, vol. 28, no. 6, pp. 739-754, 1995. 
[12] C. H. Chen, "Technical note: non-Darcy mixed convection from a horizontal surface with variable surface heat flux in a porous medium," Numerical Heat Transfer A, vol. 30, no. 8, pp. 859-869, 1996.

[13] F. C. Chou and P. Y. Chung, "Effect of stagnant conductivity on non-darcian mixed convection in horizontal square packed channels," Numerical Heat Transfer A, vol. 27, no. 2, pp. 195-209, 1995.

[14] V. Prasad, F. C. Lai, and F. A. Kulacki, "Mixed convection in horizontal porous layers heated from below," Journal of Heat Transfer, vol. 110, no. 2, pp. 395-402, 1988.

[15] C.-K. Chen and C.-H. Chen, "Nonuniform porosity and non-Darcian effects on conjugate mixed convection heat transfer from a plate fin in porous media," International Journal of Heat and Fluid Flow, vol. 11, no. 1, pp. 65-71, 1990.

[16] M. F. El-Amin, N. A. Ebrahiem, A. Salama, and S. Sun, "Radiative mixed convection over an isothermal cone embedded in a porous medium with variable permeability," Journal of Applied Mathematics, vol. 2011, Article ID 124590, 2011.

[17] E. M. A. Elbashbeshy, "The mixed convection along a vertical plate embedded in non-Darcian porous medium with suction and injection," Applied Mathematics and Computation, vol. 136, no. 1, pp. 139-149, 2003.

[18] I. A. Hassanien and T. H. Al-arabi, “Non-Darcy unsteady mixed convection flow near the stagnation point on a heated vertical surface embedded in a porous medium with thermal radiation and variable viscosity," Communications in Nonlinear Science and Numerical Simulation, vol. 14, no. 4, pp. 1366-1376, 2009.

[19] S. Jayanthi and M. Kumari, "Effect of variable viscosity on non-Darcy free or mixed convection flow on a vertical surface in a non-Newtonian fluid saturated porous medium," Applied Mathematics and Computation, vol. 186, no. 2, pp. 1643-1659, 2007.

[20] K. Khanafer and K. Vafai, "Double-diffusive mixed convection in a lid-driven enclosure filled with a fluid-saturated porous medium," Numerical Heat Transfer A, vol. 42, no. 5, pp. 465-486, 2002.

[21] K. C. Wong and N. H. Saeid, "Numerical study of non-Darcian effects on jet impingement cooling in a horizontal porous layer in the mixed convection regime," International Communications in Heat and Mass Transfer, vol. 36, no. 1, pp. 45-50, 2009.

[22] F. C. Lai, "Mixed convection in saturated porous media," in Hand Book of Porous Media, K. Vafai, Ed., pp. 605-661, Marcel Dekker, New York, NY, USA, 2000.

[23] B. V. R. Kumar, S. V. S. S. N. V. G. K. Murthy, V. Sangwan, M. Nigam, and P. Chandra, “Non-darcy mixed convection in a fluid-saturated square porous enclosure under suction effect: part I," Journal of Porous Media, vol. 13, no. 6, pp. 537-554, 2010.

[24] R. Nazar, N. Amin, D. Filip, and I. Pop, "The Brinkman model for the mixed convection boundary layer flow past a horizontal circular cylinder in a porous medium," International Journal of Heat and Mass Transfer, vol. 46, no. 17, pp. 3167-3178, 2003.

[25] N. P. Singh, A. K. Singh, and H. Sigh, "Mixed convection flow past a porous vertical plate bounded by a porous medium in a rotating system in the presence of magnetic filed," Journal of Porous Media, vol. 13, no. 7, pp. 623-633, 2010.

[26] S. Mahmud and I. Pop, "Mixed convection in a square vented enclosure filled with a porous medium," International Journal of Heat and Mass Transfer, vol. 49, no. 13-14, pp. 2190-2206, 2006.

[27] D. Srinivasacharya and Ch. RamReddy, "Mixed convection in a doubly stratified micropolar fluid saturated non-darcy porous medium," The Canadian Journal of Chemical Engineering, vol. 9999, pp. $1-10,2011$.

[28] V. Kurdyumov and A. Linan, "Free and forced convection around line sources of heat and heated cylinders in porous media," Journal of Fluid Mechanics, vol. 427, pp. 389-409, 2001.

[29] K. Vafai and H. Hadim, "Overview of current computational studies of heat transfer in porous media and their applications-natural and mixed convection," in Advances in Numerical Heat Transfer, W. J. Mikowycz and E. M. Sparrow, Eds., vol. 2, pp. 331-369, Taylor \& Francis, New York, NY, USA, 2000.

[30] M. A. Waheed, G. A. Odewole, and S. O. Algbe, "Mixed convective heat transfer in rectangular enclosures filled with porous media," ARPN Journal of Engineering and Applied Sciences, vol. 6, no. 8, pp. 47-60, 2011.

[31] N. H. Saeid and I. Pop, "Non-Darcy natural convection in a square cavity filled with a porous medium," Fluid Dynamics Research, vol. 36, no. 1, pp. 35-43, 2005.

[32] D. A. S. Rees and D. S. Riley, "Free convection above a near horizontal semi-infinite heated surface embedded in a saturated porous medium," International Journal of Heat and Mass Transfer, vol. 28, no. 1, pp. 183-190, 1985. 
[33] D. A. S. Rees and I. Pop, "Non-Darcy natural convection from a vertical wavy surface in a porous medium," Transport in Porous Media, vol. 20, no. 3, pp. 223-234, 1995.

[34] D. A. S. Rees, "The effect of inertia on free convection from a horizontal surface embedded in a porous medium," International Journal of Heat and Mass Transfer, vol. 39, no. 16, pp. 3425-3430, 1996.

[35] B. V. R. Kumar and Shalini, "Double diffusive natural convection in a doubly stratified wavy porous enclosure," Applied Mathematics and Computation, vol. 171, no. 1, pp. 180-202, 2005. 


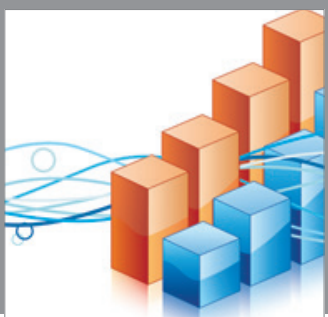

Advances in

Operations Research

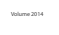



\section{The Scientific} World Journal


International Journal of

Mathematics and

Mathematical

Sciences


Journal of

Applied Mathematics


Submit your manuscripts at http://www.hindawi.com
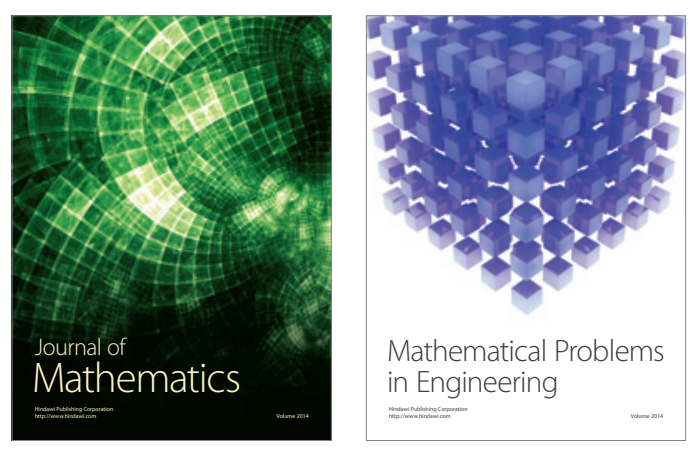

Mathematical Problems in Engineering
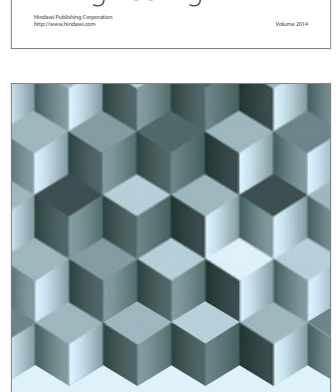

Journal of

Function Spaces


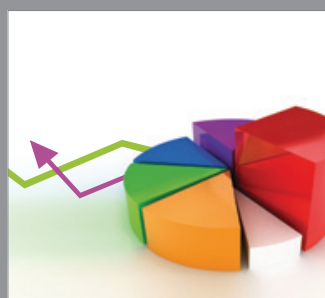

ournal of

Probability and Statistics

Promensencen
